\title{
Albugo-imposed changes to tryptophan- derived antimicrobial metabolite biosynthesis may contribute to suppression of non-host resistance to Phytophthora infestans in Arabidopsis thaliana
}

David C. Prince ${ }^{1,2}$, Ghanasyam Rallapalli, ${ }^{1,3}$, Deyang Xu4, Henk-jan Schoonbeek ${ }^{5}$, Volkan Çevik ${ }^{1,6}$, Shuta Asai ${ }^{1,7}$, Eric Kemen ${ }^{1,8}$, Neftaly Cruz-Mireles ${ }^{1}$, Ariane Kemen ${ }^{1,8}$, Khaoula Belhaj ${ }^{1}$, Sebastian Schornack ${ }^{1,9}$, Sophien Kamoun ${ }^{1}$, Eric B. Holub ${ }^{10}$, Barbara A. Halkier ${ }^{4}$ and Jonathan D. G. Jones ${ }^{1 *}$

\begin{abstract}
Background: Plants are exposed to diverse pathogens and pests, yet most plants are resistant to most plant pathogens. Non-host resistance describes the ability of all members of a plant species to successfully prevent colonization by any given member of a pathogen species. White blister rust caused by Albugo species can overcome non-host resistance and enable secondary infection and reproduction of usually non-virulent pathogens, including the potato late blight pathogen Phytophthora infestans on Arabidopsis thaliana. However, the molecular basis of host defense suppression in this complex plant-microbe interaction is unclear. Here, we investigate specific defense mechanisms in Arabidopsis that are suppressed by Albugo infection.

Results: Gene expression profiling revealed that two species of Albugo upregulate genes associated with tryptophanderived antimicrobial metabolites in Arabidopsis. Albugo laibachii-infected tissue has altered levels of these metabolites, with lower indol-3-yl methylglucosinolate and higher camalexin accumulation than uninfected tissue. We investigated the contribution of these Albugo-imposed phenotypes to suppression of non-host resistance to $P$. infestans. Absence of tryptophan-derived antimicrobial compounds enables $P$. infestans colonization of Arabidopsis, although to a lesser extent than Albugo-infected tissue. A. laibachii also suppresses a subset of genes regulated by salicylic acid; however, salicylic acid plays only a minor role in non-host resistance to $P$. infestans.

Conclusions: Albugo sp. alter tryptophan-derived metabolites and suppress elements of the responses to salicylic acid in Arabidopsis. Albugo sp. imposed alterations in tryptophan-derived metabolites may play a role in Arabidopsis nonhost resistance to $P$. infestans. Understanding the basis of non-host resistance to pathogens such as $P$. infestans could assist in development of strategies to elevate food security.
\end{abstract}

Keywords: Phytophthora infestans, Albugo, Arabidopsis thaliana, Glucosinolates, Camalexin, Salicylic acid, Non-host resistance

\footnotetext{
* Correspondence: jonathan.jones@tsl.ac.uk

${ }^{1}$ The Sainsbury Laboratory, Norwich Research Park, Norwich NR4 7UH, United Kingdom

Full list of author information is available at the end of the article
} 


\section{Background}

Plants are exposed to diverse pathogens and pests, yet most plants are resistant to most plant pathogens. Successful pathogens and pests suppress plant immunity to enable plant colonization. Current models envisage a multi-level evolutionary arms race between plants and pathogens or pests [1-4]. Plant defense responses are initiated by recognition of pathogen or pest attack via detection of pathogen molecules by plant cell surface receptors. Relatively invariant and indispensable molecules known as microbe- or pathogen-associated molecular patterns, are recognized by transmembrane pattern recognition receptors at the plasma membrane. This leads to signaling responses that result in pattern-triggered immunity (PTI). PTI is sufficient to prevent colonization by most non-adapted pathogens or pests, but pathogens which are adapted to particular host plants have evolved effectors that suppress PTI. In turn, plants evolved intracellular receptors that recognize the structure or action of effectors, resulting in effector-triggered immunity (ETI). The pathogen may subsequently adapt to the host further by evolving a variant non-recognized effector or evolving other effectors to suppress ETI.

Non-host resistance (NHR) describes the ability of all members of a plant species to successfully prevent colonization by any given member of a pathogen species $[5,6]$. In principle, NHR might result from the triggering of PTI, ETI or antimicrobial secondary metabolites. It has been proposed that the more distantly related a nonhost plant is from a host plant for a pathogen, the greater the relative contribution of PTI compared to ETI in NHR [7].

Albugo species are obligate biotrophic oomycetes that cause white blister or white rust disease in plants [8]. Albugo laibachii specializes on Arabidopsis [9], whereas A. candida is comprised of physiological races (formae speciales) that cause disease in diverse members of the Brassicaceae, Cleomaceae, and Capparaceae [8, 10]. Although most plants resist most pathogens, Albugo spp. not only overcome plant immune responses against themselves, but also suppress immunity against other filamentous pathogens. A. laibachii and A. candida can suppress resistance in Arabidopsis and Brassica juncea to downy mildews and other filamentous pathogens to which the plants are naturally resistant $[10,11]$. Suppression of immunity could allow $A$. candida strains with different host ranges to co-exist on the same host and sexually reproduce, thus allowing genetic exchange that potentially facilitates colonization of new hosts [10].

We recently found that $A$. laibachii suppresses Arabidopsis non-host resistance to Phytophthora infestans [12]. P. infestans is a hemibiotrophic oomycete that causes late blight disease in potato and tomato, leading to global yield losses [13], and is adapted to a few solanaceous plant species [14], but not to Arabidopsis [15]. A better understanding of the mechanisms that prevent $P$. infestans colonizing Arabidopsis may lead to new methods for controlling late blight disease in crop species. Crop protection strategies based on non-host resistance are of interest because they have the potential to be durable. Initial efforts to understand Arabidopsis NHR to $P$. infestans examined cytological and gene expression responses. Resistance is associated with epidermal cell death and induction of jasmonic acid (JA) responses followed by salicylic acid (SA) responses [15, 16]. However, the coronatine-insensitive 1 (coi-1) mutant, compromised in JA signaling, is resistant to $P$. infestans [17]. Subsequently, several Arabidopsis genes involved in NHR to $P$. infestans have been identified. Penetration 2 (PEN2) encodes an atypical myrosinase that hydrolyses 4-methoxyindol-3-ylmethylglucosinolate (4MO-I3M) into antimicrobial compounds [18]. PEN3 encodes a pleiotropic drug resistance ATP-binding cassette (ABC) transporter implicated in secreting antimicrobial compounds, including those produced by PEN2 [19-21]. pen 2 and pen $3 / a t p d r 8$ mutants show increased epidermal penetration and invasive growth by $P$. infestans and subsequent enhanced plant cell death in response [19, 22, 23]. A forward genetic screen to identify additional components of Arabidopsis NHR to P. infestans identified enhanced response to Phytophthora (erp) mutants $[24,25]$. erp1 encodes a phospholipid:sterol acyltransferase and shows increased cell death and callose depositions in the mesophyll without increased growth by the pathogen [24]. erp6 encodes EDR1 (enhanced disease resistance1) and plays a role in post-invasive NHR to $P$. infestans, where it acts as a negative regulator of PTI, SA signaling, and callose deposition [25]. However, while $P$. infestans can penetrate into the leaf tissue of some of the Arabidopsis mutants so far identified, there have been no reports of $P$. infestans producing haustoria or sporulating.

Compounds that are not directly involved in the primary processes of basic growth and development are termed secondary metabolites, which comprise a large collection of diverse small molecules. Specific classes of secondary metabolite are often restricted to a narrow phylogenetic lineage [26], but may perform conserved functions in plant immunity [27]. Arabidopsis secondary metabolites with a role in defense include the tryptophan-derived secondary metabolites glucosinolates, which are mostly restricted to the order Brassicales [28], and camalexin that appears to be present only in species belonging to the Camelinae tribe [29]. Camalexin and indolic glucosinolates play a role in plant immunity against diverse microbial pathogens and insect pests (reviewed by [30]). Interestingly, tryptophan-derived secondary metabolites have recently been shown to play a 
role in immunity to the oomycetes Phytophthora brassicae and Phytophthora capsici [31, 32]. The importance of camalexin to plant immunity in the Brassicales can also be seen from the examples of pathogens that detoxify these compounds in order to colonize the host [33-35].

The phenolic phytohormone SA plays an important signaling role in plant immunity [36]. SA regulates immunity, especially against biotrophs and hemibiotroph pathogens [37]. PTI and ETI lead to the accumulation of SA [38-40] and therefore the combined effects can be thought of as SA-triggered immunity (SATI). Mutants in SA signaling are more susceptible to both adapted and non-adapted pathogens (e.g. [31, 41, 42]), and effectors from several pathogen species target SA accumulation and SATI (reviewed by [43]).

The Albugo-Arabidopsis pathosystem offers the opportunity to investigate the mechanistic nature of immunesuppression in detail. We investigated how Albugo spp. suppress Arabidopsis NHR to P. infestans. We used expression profiling to look for plant pathways regulated by two Albugo species during infection. Albugo infection of Arabidopsis alters the profile of tryptophan-derived secondary metabolites, increasing camalexin accumulation and decreasing indol-3-ylmethylglucosinolate (I3M) levels. Interestingly, the camalexin accumulated in Albugo-infected tissue, though detectable in extracts, appears to be biologically unavailable for defense against the necrotrophic fungus Botrytis cinerea. Albugo also suppresses SATI, but lack of SA is not sufficient to allow colonization of Arabidopsis by $P$. infestans. Our results therefore suggest that Albugo affects many aspects of plant immunity, leading to the plant becoming susceptible to previously resisted pathogens, and that tryptophan-derived metabolites play a role in Arabidopsis NHR to $P$. infestans.

\section{Methods \\ Biological material}

Arabidopsis (Arabidopsis thaliana) plants were grown as previously described [12]. Seeds were sown on Scotts Levington F2 compost (Scotts, Ipswich, UK) and vernalized for one week at $5-6{ }^{\circ} \mathrm{C}$. Seedlings were subsequently grown in a controlled environment room (CER) with a $10 \mathrm{~h}$ day and a $14 \mathrm{~h}$ night photoperiod and at a constant temperature of $22{ }^{\circ} \mathrm{C}$ for 2 weeks and then pricked out into "Arabidopsis mix" (600 L F2 compost, $100 \mathrm{~L}$ grit, $200 \mathrm{~g}$ Intercept insecticide) and returned to the CER. Arabidopsis plants were infected with Albugo when they were 4 or 5 weeks old. Arabidopsis lines used in this study are listed in Additional file 1.

Brassica juncea seeds were sown on Scotts Levington F2 compost (Scotts). Seedlings were subsequently grown in a CER with a $10 \mathrm{~h}$ day and a $14 \mathrm{~h}$ night photoperiod and at a constant temperature of $22{ }^{\circ} \mathrm{C}$ for 1 week and then pricked out into "Arabidopsis mix" and returned to the CER.

Phytophthora infestans isolate 88069td expresses a cytosolic tandem DsRed protein [44]. P. infestans isolate NL12226 was isolated by Geert Kessel (Wageningen University and Research, Wageningen) in 2012 from infected Solanum tuberosum cultivar Toluca in Valthermond, Flevoland, The Netherlands. Both isolates were cultured on rye sucrose agar [45] at $18{ }^{\circ} \mathrm{C}$ in the dark [46].

Albugo strains were propagated as follows: zoosporangia from plants inoculated 14 days earlier were suspended in cold water and incubated on ice for $30 \mathrm{~min}$. The spore suspension was then sprayed on plants using a spray gun, and plants were incubated in a cold room $\left(5{ }^{\circ} \mathrm{C}\right)$ in the dark overnight to promote Albugo spore germination. Infected plants were kept under 10 -h light and 14-h dark cycles with a $21{ }^{\circ} \mathrm{C}$ day and $14{ }^{\circ} \mathrm{C}$ night temperature. Albugo laibachii strain Nc14 [47] was maintained on Col-gl resistance to powdery mildew (RPW)8.1 and RPW8.2 Arabidopsis [48]. Albugo candida (Ac) strains Ac2V [10], AcEx1 (this study), and AcNc2 [10] were maintained on Brassica juncea cultivar Burgonde, Col-0, and Ws-2 Arabidopsis ecotypes, respectively.

Hyaloperonospora arabidopsidis isolate Waco9 was inoculated as described previously $[49,50]$.

Botrytis cinerea was cultured and inoculated as described previously [51]. B05.10 is the wildtype strain. $\triangle \mathrm{BcatrB} 4$ is a B05.10 derived gene-replacement mutant in BcatrB [52]. The BcatrB promoter- $\beta$-Glucuronidase (GUS) fusion strain BcatrBp803GUS-7 contains the 803 bp upstream of the BcatrB start codon fused in-frame to the uidA gene from Escherichia coli [53]. The OliCpromoter-GUS fusion strain OliCGUS shows constitutive expression of the uidA gene [53, 54].

\section{Gene expression analysis over Albugo infection time course}

To harvest samples representing a time course of infection of A. laibachii and A. candida on Arabidopsis we have used a multi-parent recombinant inbred derived line, Multi-parent Advanced Generation Inter-Cross (MAGIC) 107 [55]. Arabidopsis ecotype Col-0 is resistant to AcNc2 and ecotype Ws-2 shows necrotic lesions, while MAGIC 107 shows significantly reduced trailing necrosis and exhibits a compatible interaction with AcNc2 and AlNc14. AcNc2 and AlNc14 were spray inoculated as described above. For mock treatment, plants were sprayed with cold water. Plants were incubated overnight in the dark at $5{ }^{\circ} \mathrm{C}$. Arabidopsis leaf samples were collected immediately after the cold treatment $(0$ time point) and at 2, 4, 6, and 8 days post inoculation (dpi). Four independent biological replicates for each treatment and each time point were collected. 
RNA extraction, EXpression Profiling through Randomly Sheared cDNA tag Sequencing (EXPRSS) library preparation for Illumina sequencing, and sequence read to gene mapping were performed as described previously [56]. Double stranded cDNA samples were sheared for library preparation using Covaris S220X (Covaris settings: intensity, 5; duty cycle, 20\%; cycles/burst, 200; duration, $60 \mathrm{sec}$ ). The libraries were sequenced using Illumina Genome Analyzer II producing sequence reads of 76 nucleotides. The sequence data has been deposited in the National Center for Biotechnology Information's Gene Expression Omnibus [57] and are available under series accession number GSE75016. Sequence reads to gene associations were carried out using the considerations and scripts previously published [56]. Mock samples were analyzed in pairwise manner with each Albugo species infection data, independently. Quality-filtered libraries of mock and AlNc14-infected samples were aligned to the combined genomes of The Arabidopsis Information Resource version 10 (TAIR10) [58] and AlNc14 version 1 [47]; similarly, mock and AcNc2-infected samples were aligned to combined genomes of TAIR10 and AcNc2 version 1 [10] using Bowtie version 0.12.8 [59]. Unaligned reads from previous steps were mapped to the combined cDNA reference sequences of the respective Arabidopsis (TAIR10) and Albugo strain (AlNc14 version1 and AcNc2 version1) combinations using Novoalign v2.08.03 [60]. Details of software parameters, genomes, and gene sequences used for the analysis are available online [61].

Uniquely aligned read counts were selected for differential expression analysis. For gene expression analysis, each Albugo (AlNc14 or AcNc2) infection time point data was compared against respective mock time point data resulting from pairwise analysis. Differential expression analysis was performed using the $\mathrm{R}$ statistical language [62] version 2.11.1 with the Bioconductor package [63] and edgeR version 1.6.15 [64] with the exact negative binomial test using tagwise dispersions. The Benjamini-Hochberg method [65] based false discovery rate (FDR) was applied and genes with FDR $<0.01$ were selected as differentially expressed (Additional file 2).

For comparative analysis of benzo-(1,2,3)-thiadiazole-7carbothioic acid (BTH) and JA responsive gene progression during Albugo infection, previously published microarray data of Arabidopsis treatment with BTH [66] and methyl jasmonate $[67,68]$ were used. Microarray data normalization and differential expression analysis was carried out as described previously [56]. Genes with FDR $<0.05$ were selected for comparative gene expression analysis.

\section{Gene Ontology (GO) enrichment analysis}

Lists of Arabidopsis genes that were up-regulated or down-regulated at each time point in infected plant tissue compared to the control were compiled (Additional file 3). Overlap between the AlNc14 and AcNc2 gene lists was determined using the Venn diagram available in the Public Research Centre for Health [69]. These lists were then used to perform Singular Enrichment Analysis with FDR $=0.05$ using AgriGO v1.2 and default settings [70]. GO annotations are based on TAIR10.

\section{P. infestans infection assays}

Sequential infection of plants with Albugo and then $P$. infestans were carried out with appropriate controls as previously described [12].

Assays with non-Albugo-infected Col-0 and mutant Arabidopsis were conducted by placing droplets of $P$. infestans spores on the abaxial side of detached leaves and incubating for up to 3 days at $100 \%$ relative humidity. After 36 hours, the droplets were gently removed using paper towel to prevent the growth of $P$. infestans in the water rather than the leaf.

\section{Visualizing and quantifying $P$. infestans}

P. infestans 88069td colonization of Arabidopsis was visualized using a Leica M165FC microscope with DFC425 camera and EL6000 light source (Leica Microsystems, Milton Keynes, UK) and a DSR filter (excitation wavelength of 510-560 $\mathrm{nm}$ and emission wavelength of $590-650 \mathrm{~nm})$. P. infestans growth is represented by red fluorescence. Leaves that were inoculated with $P$. infestans on the abaxial surface may show no fluorescence from the adaxial surface due to lack of pathogen colonization (e.g. Col-0 plants).

P. infestans colonization of Arabidopsis was quantified using qRT-PCR. Leaf discs (10 $\mathrm{mm}$ diameter) were punched out of Arabidopsis leaves inoculated with $P$. infestans and DNA extracted with DNeasy plant mini kit (Qiagen, Hilden, Germany). Four discs were used per replicate for water-sprayed plants, and three discs per replicate for Albugo-sprayed plants. DNA was diluted to $5 \mathrm{ng} / \mu \mathrm{L}$ and $5 \mu \mathrm{L}$ used per qRT-PCR reaction. qRT-PCR was conducted as described below, using primers for At3g21215 and PiO8-3-3 (Additional file 4) to compare the amount of $P$. infestans DNA present.

$P$. infestans NL12226 sporulation on Col-0 and cyp $79 b 2 / b 3$ Arabidopsis was quantified by infecting leaves from 4-week-old plants (as described above), then checking for the presence of $P$. infestans spores between 3 and 5 dpi by placing droplets of water on the leaf surface and examining them under a light microscope.

\section{qRT-PCR of plant genes}

Plants were sprayed with Albugo or water, and subsequently inoculated with $P$. infestans as described above. Samples consisted of two Arabidopsis leaves and two samples were taken per experiment per time point, with the experiment being repeated three times. 
Samples were homogenized using a TissueLyser II (Qiagen) and 3-mm tungsten carbide beads (Qiagen) under cold conditions. Total RNA was extracted using TriReagent (Sigma-Aldrich), Direct-zol ${ }^{\mathrm{TM}}$ RNA miniprep kit (Zymo Research, Irvine, CA), and on-column DNase treatment. Purity and integrity were checked using a Nanodrop 8000 (Thermo Scientific) and agarose gel. cDNA was synthesized from $1 \mu \mathrm{g}$ RNA using Oligo $\mathrm{dT}_{12-18}$ primers (Life Technology, Paisley, UK) and Superscript III reverse transcriptase (Life Technology) according to the manufacturer's instructions. cDNA from these reactions was diluted 1:20 with distilled water before qRT-PCR. Stable reference genes for normalization were selected as previously described [71]. Candidate reference genes were selected from previously identified superior reference genes [72] (Additional file 4). Analysis of eight candidates (elongation factor 1 alpha, two $A$ and related phosphatase-associated protein42-interacting protein of $41 \mathrm{kD}$ (TIP41), U-BOX, glyceraldehyde-3-phosphate dehydrogenase C2, ACTIN2, PEROXIN4, monensin sensitivity1, and adaptor protein-2 $M U$-ADAPTIN) using geNORM [73] and NormFinder [74] identified the optimal number of reference genes needed for normalization to be two, and the two most stable genes across the experimental conditions to be TIP41 (At4g34270) and elongation factor 1-alpha (At5g60390). Primer sequences and annealing temperature used for qRT-PCR are described in Additional file 4.

\section{qRT-PCR assays}

Each reaction consisted of $20 \mu \mathrm{L}$ containing $5 \mu \mathrm{L}$ of DNA or cDNA and $0.5 \mu \mathrm{M}$ of each primer (Additional file 4) added to SYBR Green JumpStart Taq ReadyMix (SigmaAldrich) in a single well of a 96-well white ABgene PCR plate (Thermo Scientific). Reactions were run in a CFX96 Real-Time System with a C1000 Thermal Cycler (BioRad). PCRs were carried out using the following thermocycle: $3 \mathrm{~min}$ at $95^{\circ} \mathrm{C}$, followed by 40 cycles of $30 \mathrm{~s}$ at $95^{\circ} \mathrm{C}$, $30 \mathrm{~s}$ at the relevant annealing temperature (Additional file 4), and $30 \mathrm{~s}$ at $72{ }^{\circ} \mathrm{C}$, followed by melt curve analysis (65-95 ${ }^{\circ} \mathrm{C}$ at $0.5{ }^{\circ} \mathrm{C}$ increments, $5 \mathrm{~s}$ for each). Primer efficiencies were calculated using a dilution series of DNA or cDNA. To calculate the relative expression levels of target genes, mean cycle threshold values for each sample-primer pair combination were calculated from three replicate reaction wells. The cycle threshold values and primer efficiencies were then used to calculate normalized relative quantities (NRQs) for each gene using the EasyqpcR package [75] in R. NRQs were then $\log _{2}$ transformed [76] and statistical analyses performed as described below.

\section{Metabolite analysis}

Plants were sprayed with Albugo or water, and subsequently inoculated with $P$. infestans or water as described above. Single leaves were collected 20 hours post
P. infestans/control treatment for analysis of indolic glucosinolates and 48 hours post treatment for camalexin analysis.

Plants were sprayed with AlNc14 or water, and subsequently sprayed with $B$. cinerea or quarter-strength potato dextrose broth. Sets of three leaves were collected 26 hours post B. cinerea/control treatment for camalexin analysis. All samples were immediately flash frozen in liquid nitrogen and subsequently dry frozen.

Glucosinolates were analyzed as desulfo glucosinolates through a modified version of a previously described method [77]. Leaf material was lyophilized and homogenized in $85 \%$ methanol containing $0.02 \mathrm{mM}$ para-hydroxybenzyl glucosinolate as internal standard. Samples were centrifuged at $13,000 \mathrm{~g}$ for $10 \mathrm{~min}$ and the supernatant was transferred to a 96-well filter plate (Millipore) loaded with $45 \mathrm{mg}$ diethylaminoethyl sephadex ${ }^{\mathrm{TM}}$ A-25 column material (GE Healthcare Biosciences), which had been equilibrated for 4 hours in $300 \mu \mathrm{L}$ water before samples were applied. Glucosinolates were bound to the column material while samples were sucked through the filter plate by applying a brief vacuum. Afterwards, columns were washed with $2 \times 100 \mu \mathrm{L} 70 \%$ methanol and $2 \times 100 \mu \mathrm{L}$ water, respectively. Then, 20 $\mu \mathrm{L}$ sulfatase (SIGMA E.C. 3.1.6.) solution $\left(2 \mathrm{mg} \mathrm{mL}^{-1}\right)$ was added to the columns and allowed to incubate at room temperature overnight; $100 \mu \mathrm{L}$ water were applied to the columns and a short spin eluted the desulfoglucosinolates into a 96-well format plate. The samples were analyzed on a Shimadzu high performance liquid chromatography (HPLC)-DAD system and separated on a Zorbax SB-AQ column $(4.6 \mathrm{~mm} \times 25 \mathrm{~cm}, 5 \mu \mathrm{m}$ particle size) at a flow rate of $1 \mathrm{~mL} \mathrm{m^{-1 }}$. Compounds were detected at $229 \mathrm{~nm}$ using a diode array UV and separated utilizing eluents (A: $\mathrm{H}_{2} \mathrm{O}, \mathrm{B}: 100 \%$ acetonitrile) using the following program: 5 min gradient from $1.5 \%$ to $7 \%$ eluent B; 5 min gradient from $7 \%$ to $25 \%$ eluent B; 4 min gradient from $25 \%$ to $80 \%$ eluent $\mathrm{B} ; 3 \mathrm{~min}$ at $80 \%$ eluent B; 2 min gradient from $80 \%$ eluent B to $35 \%$ eluent B; 2 min gradient from $35 \%$ to $1.5 \%$ eluent B; a final $3 \mathrm{~min}$ at $1.5 \%$ eluent $\mathrm{B}$. Response factors for absorbance at $229 \mathrm{~nm}$ were used to quantify the desulfoglucosinolates [78-80].

Leaf samples for camalexin analysis were disrupted in methanol using a Retsch Mixer Mill 303 (Retsch, Haan, Germany). Samples were spun down and the supernatant collected, and the process was repeated with the pellet tissue. Supernatants were filtered through a 0.22$\mu \mathrm{m}$ filter (Millipore). Samples were quantified using synthetic camalexin as an external standard. The peak at 5.17 min was identified as camalexin by comparison with authentic standard with respect to retention time and UV spectrum (photodiode array detector 168, Beckman Instruments, Fullerton, CA) and quantified by 
using a Shimadzu F-10AXL fluorescence detector (318 $\mathrm{nm}$ excitation and $370 \mathrm{~nm}$ emission) and by UV absorption at $318 \mathrm{~nm}$.

\section{Botrytis cinerea}

Inoculation of Arabidopsis with B. cinerea was performed as described previously [81], with minor modifications. For disease assays, plants sprayed with AlNc14 or water 12 days previously were pairwise-inoculated with the different isolates using $5 \mu \mathrm{L}$ droplets of $2.5 \times 10^{5}$ spores per $\mathrm{mL}$ in quarter-strength potato dextrose broth. Six leaves per plant and at least eight plants per experiment were used. Lesion diameters were measured at $3 \mathrm{dpi}$.

For determination of GUS activity in OliCGUS and BcatrBp803GUS-7 water- or AlNc14-sprayed leaves were inoculated by pairwise droplet inoculation of three droplets of each strain on either side of the leaf or sprayed as a whole plant till near run-off. For visual examination of the droplets inoculated leaves were detached at 48 hours post inoculation (hpi) and vacuum-infiltrated three times for 2 mins in X-Gluc staining buffer $(50 \mathrm{mM}$ sodium phosphate buffer $\mathrm{pH} 7.0,10 \mathrm{mM}$ ethylenediaminetetraacetic acid (EDTA), $0.5 \mathrm{mM} \mathrm{K}_{3} \mathrm{Fe}(\mathrm{CN})_{6}, 0.5 \mathrm{mM}$ $\mathrm{K}_{4} \mathrm{Fe}(\mathrm{CN})_{6}, 0.5 \% \mathrm{w} / \mathrm{v}$ Triton $\mathrm{X}-100$ and $0.5 \mathrm{mg} \mathrm{mL}^{-1} \mathrm{X}$ Gluc cyclohexylammonium salt) [51, 82]. Leaves were incubated for $20 \mathrm{~h}$ at $37{ }^{\circ} \mathrm{C}$, destained in four changes of ethanol, and the intensity of blue staining at each inoculation site was estimated on a scale from 0 to 3 . The score of all droplets per leaf was averaged and expressed as percentage of the maximum per leaf and data presented are averages of three experiments with at least five leaves per pairwise comparison. For determination of GUS activity in sprayed leaves, three leaves were collected $48 \mathrm{hpi}$, blotted dry on tissue paper, weighed and frozen in $2-\mathrm{mL}$ tubes. Leaves in each tube were pulverised in a genogrinder 2010 [83] with two 3-mm stainless steel balls for $1 \mathrm{~min}$ at 1250 strokes per minute in blocks cooled with dry-ice. Enzymes were extracted with $25 \mathrm{mM}$ sodium phosphate buffer $\mathrm{pH} 7.0$ with $0.1 \%$ Triton and GUS activity determined as the conversion of 4methylumbelliferyl- $\beta$-D-glucuronide (Sigma) by GUS to its fluorescent degradation product on a Varioskan Flash multiplate reader (Thermo Scientific) adapted from Jefferson et al. [84]. The remaining pellet was used for total DNA extraction and qRT-PCR determination of $B$. cinerea levels in each sample according to Gachon et al. [85] (Additional file 4). GUS expression was normalized against the $B$. cinerea weight portion of each sample.

\section{Microscopy of $P R 1::$ GUS leaves}

GUS activity in leaves of pathogenesis-related 1 (PR1)::GUS plants was assayed histochemically with 5bromo-6-chloro-3-indolyl b-D-glucuronide cyclohexylammonium salt $\left(1 \mathrm{mg} \mathrm{mL}^{-1}\right.$ ) (Magenta b-D-GlcA CHX,
Carbosynth Limited, Compton, UK) in a buffer containing $100 \mathrm{mM}$ sodium phosphate $\mathrm{pH}$ 7.0, $0.5 \mathrm{mM}$ potassium ferrocyanide (Sigma-Aldrich, St Louis, USA), $0.5 \mathrm{mM}$ potassium ferricyanide (Sigma-Aldrich), $10 \mathrm{mM}$ EDTA (Thermo Scientific, Loughborough, UK), and 0.1\% Triton (Sigma-Aldrich). Arabidopsis leaves were vacuuminfiltrated with staining solution and incubated overnight at $37^{\circ} \mathrm{C}$ in the dark. Leaves were then boiled in lactophenol containing $0.17 \mathrm{mg} \mathrm{mL}^{-1}$ trypan blue (Sigma-Aldrich) for $1 \mathrm{~min}$ and destained by incubation in $2.5 \mathrm{~g} \mathrm{~mL}^{-1}$ chloral hydrate (Sigma-Aldrich). Staining of whole leaves was visualized using a Leica M165FC microscope with DFC425 camera and EL6000 light source (Leica Microsystems). The percentage of the leaf stained with magentaGlcA was determined by measuring the leaf area and the stained area using ImageJ [86].

\section{Statistical analyses}

Statistical analyses were conducted using R 3.2.2 [62] within RStudio 0.99.483 [87] (data are available in Additional files relating to each figure; please see below). Technical replicates consist of readings from the same condition in the same experiment, whereas biological replicates consist of independent experiments with batches of plants sown on different days. Data were analyzed using the following pipeline: data were assessed for their suitability to be analyzed using parametric tests by testing for the normal distribution of the residuals (D'Agostino-Pearson and Shapiro-Wilk tests) and visualizing residuals with $\mathrm{Q}-\mathrm{Q}$ plots. The assumption of equal variances between the conditions was tested using the Bartlett test for data with normally distributed residuals and the Fligner test for data with non-normally distributed residuals. If the data were suitable for conducting parametric tests, then Welch's two sample t-test or analysis of variance (ANOVA) were used as appropriate. Percentage data in Additional file 5 were transformed in order to meet the assumptions of parametric tests. The percentage of leaf stained was first arcsine square root transformed (arcsine(square root(percentage/100))), and then subsequently $\log _{10}$ transformed $\left(\log _{10}(\right.$ transformed data point +1$\left.)\right)$. If the data were not suitable for parametric tests, then the appropriate non-parametric test (Wilcoxon rank sum test, KruskalWallis rank sum test) was used if possible. Data that did not meet the assumptions for parametric tests but had more than one set of treatments were analyzed within a generalized linear model (GLM) using a Poisson distribution, or a quasi-Poisson distribution if the data were over dispersed. Multiple comparisons were corrected for using Tukey's honest significant difference (HSD) where appropriate, and otherwise Bonferroni correction. 


\section{Results}

Two Albugo species compromise plant immunity and enables sporulation of Phytophthora infestans

We recently reported that A. laibachii Nc14 (AlNc14) [47] suppresses Arabidopsis NHR to P. infestans ([12], Fig. 1a, b, d and e). As immunosuppression was also demonstrated for the related species $A$. candida $[10,11]$, we investigated whether $A$. candida infection of Arabidopsis and Brassica juncea compromises NHR to $P$. infestans. A. candida isolate Exeter 1 (AcEx1), which is adapted to many Arabidopsis ecotypes including Col-0, suppressed
NHR in Arabidopsis to P. infestans (Fig. 1c and f). A. candida isolate $2 \mathrm{~V}$ (Ac2V) is adapted to $B$. juncea but not Arabidopsis ecotypes [10], and also suppresses plant NHR to P. infestans on B. juncea (Fig. 1g-i). P. infestans sporulates in both AcEx1- and Ac2V- infected leaves (Fig. 1c, f, $\mathrm{g}$ and $\mathrm{i})$. To test if the NHR suppression was imposed by other biotrophic oomycetes that infect Arabidopsis, we inoculated Hyaloperonospora arabidopsidis (Hpa)-infected Arabidopsis with $P$. infestans. We saw no P. infestans colonization of Arabidopsis infected with the compatible $\mathrm{Hpa}$ isolate Waco9 (Additional file 6). Together, these

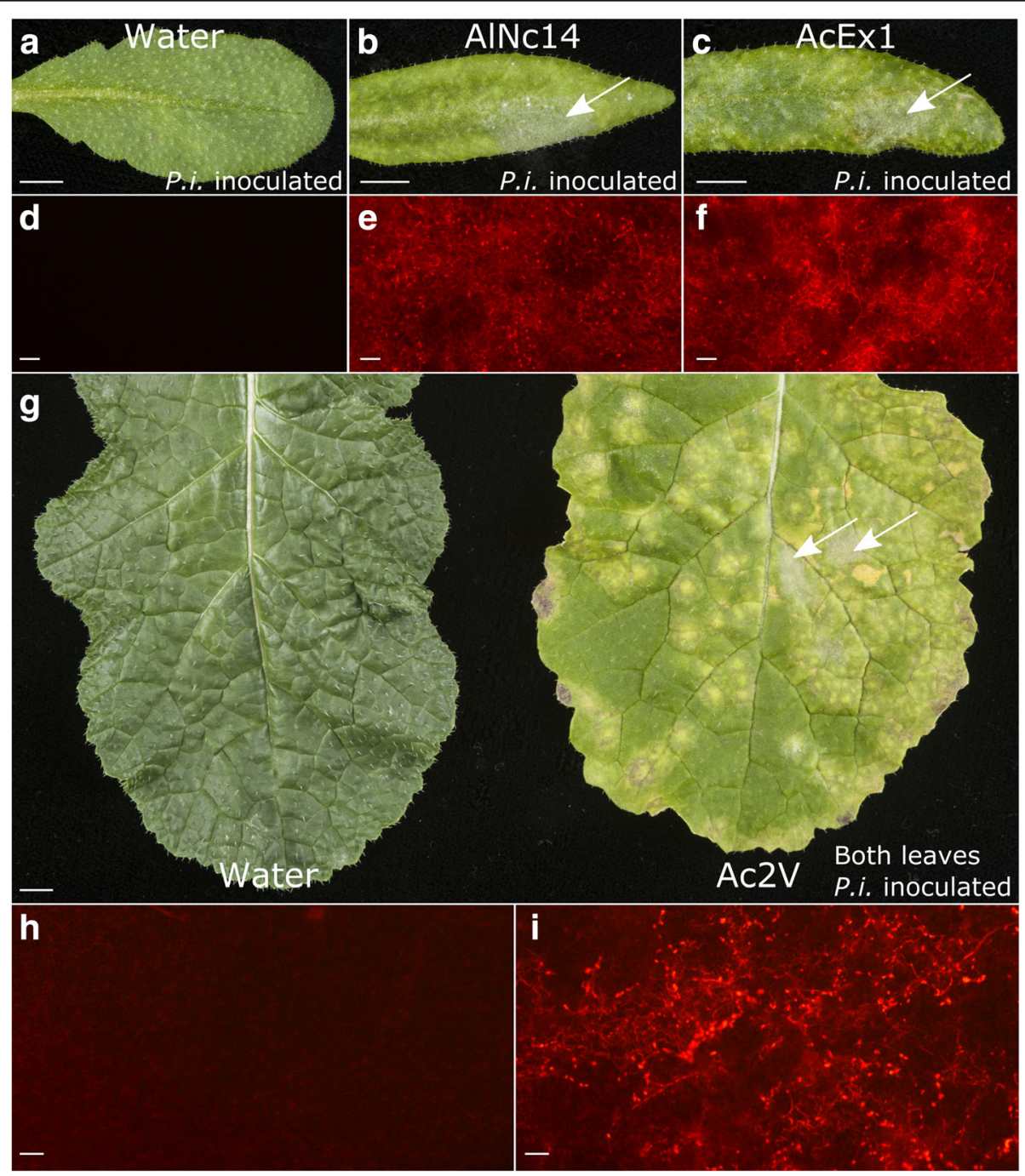

Fig. 1 Two Albugo species compromise plant immunity and enable sporulation of Phytophthora infestans. a-f Albugo species compromise Arabidopsis immunity to $P$. infestans. Water-sprayed (a, d), Albugo laibachii Nc14-sprayed (b, e), and Albugo candida AcEx1-sprayed (c, f) Col-0 leaves ( 13 days post inoculation (dpi)) were drop inoculated with $100 \mathrm{\mu L}$ of $5 \times 10^{4}$ spores per $\mathrm{mL} P$. infestans 88069 td. a-c Photographs taken 3 dpi with $P$. infestans. Scale bar: $5 \mathrm{~mm}$. Arrows denote $P$. infestans sporulation. $\mathbf{d}-\mathbf{f}$ Fluorescence microscopy of the adaxial surface of the leaf. Red fluorescence denotes $P$. infestans growth. Scale bar: $200 \mu \mathrm{m}$. Results shown are representative of three independent experiments. $\mathbf{g}-\mathbf{i} A$. candida compromises Brassica juncea immunity to $P$. infestans. g Water-sprayed (left) and A. candida Ac2V-infected (right) B. juncea leaves (12 dpi) were drop inoculated with several $250 \mu \mathrm{L}$ drops of $4 \times 10^{4}$ spores per $\mathrm{mL}$ P. infestans $88069 \mathrm{td}$. Photographs were taken $3 \mathrm{dpi}$ with P. infestans. Scale bar: $5 \mathrm{~mm}$. Arrows denote $P$. infestans sporulation. $\mathbf{h}$, i Fluorescence microscopy of the adaxial surface of water-sprayed (h) and Ac2V-infected (i) leaves. Red fluorescence denotes $P$. infestans growth. Scale bar: $200 \mu \mathrm{m}$. Results shown are representative of three independent experiments 
data suggest that suppression of NHR to $P$. infestans is imposed after infection by Albugo species but not by other biotrophic oomycete pathogens of Arabidopsis.

\section{Albugo-infection upregulates plant tryptophan metabolism}

To understand the effect of Albugo infection on plant gene expression over a time course of infection we used EXPRSS, a sensitive, reliable, and high-throughput tagbased expression profiling method [56]. We wished to compare the Arabidopsis gene expression responses to infection with two Albugo species, AlNc14 and A. candida isolate Nc2 (AcNc2). While AlNc14 is compatible with many Arabidopsis ecotypes, Col-0 is resistant to AcNc2 and Ws-2 shows necrotic lesions upon AcNc2 infection. Arabidopsis MAGIC line 107 [55] was chosen after screening multiple MAGIC lines because it shows the most compatible interaction (significantly reduced trailing necrosis) with AcNc2, and also showed compatibility with AlNc14. We hypothesized that both species of Albugo suppress NHR to P. infestans by similar mechanisms. We treated MAGIC line 107 [55] with AlNc14, AcNc2 [10], or water as a control, and then took leaf samples for RNA extraction at $0,2,4,6$, and 8 dpi. EXPRSS libraries were prepared from the extracted RNA and sequenced using Illumina sequencing. The sequences were mapped to genes, and differential expression analysis conducted. There was some overlap in the Arabidopsis genes differentially regulated by both pathogen species, with around $25 \%$ of the total up-regulated and downregulated genes across the time course shared by the two pathogen species (Fig. 2). To identify which plant pathways

specific
Shared
AcNc2
specific
Fig. $\mathbf{2}$ Genes differentially expressed in expression profiling experiment.
The number of differentially expressed genes in MAGIC 107 Arabidopsis
infected with AlNc14 or AcNc2 was calculated over an 8-day time course.
The data are the average of four experiments. The Venn diagrams show
the percentage of genes (with number of genes in brackets) that were
up-regulated (red rings) or down-regulated (blue rings) at that time point
and whether they were either unique to infection with one pathogen
species, or were shared between the two pathogen species. Combined
time points show genes that were up-regulated at one or more time
points and not subsequently down-regulated (and vice versa)

were altered by Albugo, we conducted GO enrichment analysis using AgriGo [70] on lists of differently expressed genes (Additional files 7 and 8), focusing on specific lower level terms within biological processes. Few plant pathways were up-regulated at early time points in both infections (Table 1). At later time points, pathways associated with plant defense, such as SA and JA, were up-regulated. The only enriched down-regulated plant processes shared by infection with either pathogen were photosynthesis and RNA elongation. We focused on the up-regulation of the tryptophan-derived secondary metabolites, which include camalexin and indole-derived compounds, as these pathways were enriched in genes up-regulated by AlNc14 and AcNc2 infection (Table 1; 8 dpi and Combined time points), and they have been shown to play a role in Arabidopsis immunity to other Phytophthora species [31, 32].

\section{Albugo infection changes the proportions of camalexin and indolic glucosinolates}

To explore whether tryptophan-derived secondary metabolites are involved in Arabidopsis responses to P. infestans and how Albugo infection may alter their accumulation, we measured Arabidopsis transcriptional responses and metabolite accumulation in water-sprayed and Albugo-infected plants in response to $P$. infestans. We selected genes that were at the start of the pathway (cytochrome P450 (CYP)79B2), on the camalexin branch (CYP71A13 and phytoalexin deficient3 (PAD3)), on the core indolic glucosinolate pathway (CYP83B1 and sulfotransferase16 (SOT16)), and involved in indolic glucosinolate modification (CYP81F2) (Fig. 3). At 6 hours (Fig. 4a, Additional files 9 and 10), Albugo infection alone up-regulated CYP71A13, $P A D 3$, and CYP81F2. P. infestans infection alone upregulated all of the genes except CYP83B1. SOT16 expression induced by $P$. infestans was suppressed in the presence of Albugo. At 48 hours (Fig. 4b, Additional files 10 and 11), Albugo infection alone up-regulated the same genes as at 6 hours plus CYP79B2. P. infestans infection alone upregulated the same genes as at 6 hours, with the exception of SOT16. Albugo and P. infestans infection together led to increased expression of CYP79B2 and CYP81F2, and decreased expression of CYP83B1 compared to $P$. infestans infection alone. These data support the inference of the expression profiling and GO enrichment analysis that genes involved in tryptophan-derived secondary metabolite processes are up-regulated in Albugo-infected tissue. They also show that these genes respond to $P$. infestans infection.

We measured camalexin and indolic glucosinolate (I3M and 4MO-I3M) levels in leaves with the same experimental design as above. Albugo-treatment $(\mathrm{t}=-6.037, P<0.001$, GLM) and $P$. infestans inoculation $(\mathrm{t}=-7.340, P<0.001)$ led to significant accumulation of camalexin (Fig. 4c, Additional file 10). Albugo-infected tissue accumulates significantly less $\mathrm{I} 3 \mathrm{M}(\mathrm{t}=5.884, P<0.001, \mathrm{GLM})$ but $P$. 
Table 1 Gene ontology (GO) terms enriched in Arabidopsis genes differentially expressed by both pathogen infections

\begin{tabular}{|c|c|c|c|c|c|}
\hline Category & $2 \mathrm{dpi}$ & $4 \mathrm{dpi}$ & $6 \mathrm{dpi}$ & $8 \mathrm{dpi}$ & Combined time points \\
\hline $\begin{array}{l}\text { Up-regulated vs. } \\
\text { control (0 dpi) }\end{array}$ & $\begin{array}{l}\text { - Golgi } \\
\text { apparatus }\end{array}$ & $\begin{array}{l}\cdot \text { rRNA } \\
\text { modification }\end{array}$ & $\begin{array}{l}\text { - Jasmonic acid-mediated } \\
\text { signaling pathway } \\
\text { - MAPKKK cascade } \\
\text { - Negative regulation of } \\
\text { programmed cell death } \\
\text { - Salicylic acid-mediated } \\
\text { signaling pathway } \\
\text { - Systemic acquired resistance }\end{array}$ & $\begin{array}{l}\text { - Indole derivative } \\
\text { biosynthetic processes } \\
\text { - Jasmonic acid-mediated } \\
\text { signaling pathway } \\
\text { - MAPKKK cascade } \\
\text { - Negative regulation of } \\
\text { programmed cell death } \\
\text { - Response to hormone } \\
\text { stimulus } \\
\text { - Salicylic acid-mediated } \\
\text { signaling pathway } \\
\text { - Systemic acquired } \\
\text { resistance } \\
\text { - Tryptophan metabolic } \\
\text { processes }\end{array}$ & $\begin{array}{l}\text { - Camalexin biosynthetic } \\
\text { processes } \\
\text { - Indole-derived metabolic } \\
\text { processes } \\
\text { - Jasmonic acid-mediated } \\
\text { signaling pathway } \\
\text { - MAPKKK cascade } \\
\text { - Negative regulation of } \\
\text { defense response } \\
\text { - Negative regulation of } \\
\text { programmed cell death } \\
\text { - Response to hormone } \\
\text { stimulus } \\
\text { - Salicylic acid-mediated } \\
\text { signaling pathway } \\
\text { - Systemic acquired } \\
\text { resistance } \\
\text { - Tryptophan metabolic } \\
\text { processes }\end{array}$ \\
\hline $\begin{array}{l}\text { Down-regulated vs. } \\
\text { control (0 dpi) }\end{array}$ & $\begin{array}{l}\text { - Photosynthesis } \\
\text { - RNA } \\
\text { elongation }\end{array}$ & $\begin{array}{l}\text { - Photosynthesis } \\
\text { - RNA } \\
\text { elongation }\end{array}$ & $\begin{array}{l}\text { - Photosynthesis } \\
\text { - RNA elongation }\end{array}$ & $\begin{array}{l}\text { - Photosynthesis } \\
\text { - RNA elongation }\end{array}$ & $\begin{array}{l}\text { - Photosynthesis } \\
\text { - RNA elongation }\end{array}$ \\
\hline
\end{tabular}

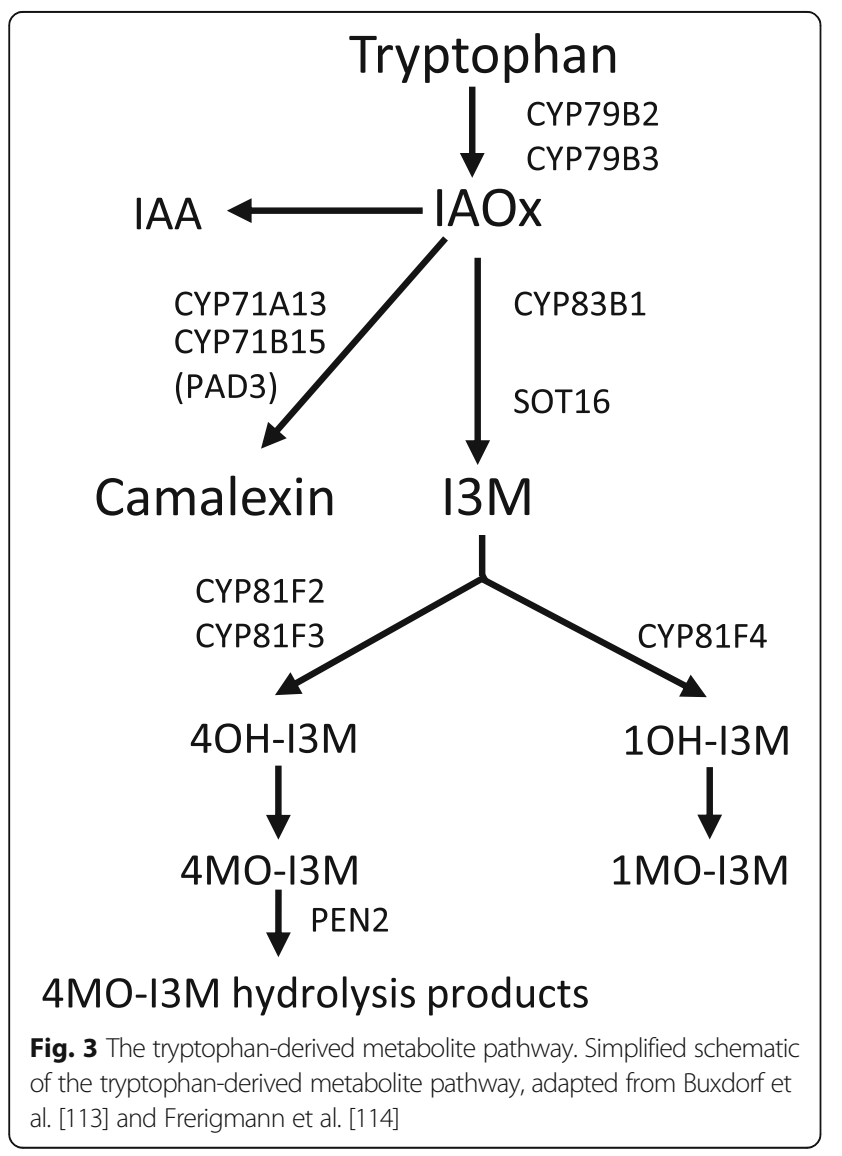

infestans inoculation has no effect $(\mathrm{t}=0.037, P=0.971)$ (Fig. 4d, Additional file 10). Neither of the treatments change the accumulation of 4MO-I3M (Albugo: $\mathrm{t}=-0.123$, $P=0.90$, P. infestans: $\mathrm{t}=-0.762, P=0.45$, GLM) (Fig. $4 \mathrm{~d}$, Additional file 10). 4MO-I3M accumulates in the pen2-1 mutant upon challenge with flg22 or non-host pathogens due to reduced hydrolysis $[18,88]$. However, we found similar results to $\mathrm{Col}-0$ when we repeated the experiment in the pen2-1 mutant (Additional files 12 and 13). In conclusion, $P$. infestans infection of Arabidopsis elicits transcriptional responses in the camalexin and indolic glucosinolate metabolic pathways, and the accumulation of camalexin. Albugo-infection appears to alter tryptophan-derived secondary metabolite levels leading to increased accumulation of camalexin and decreased accumulation of I3M.

\section{Indole glucosinolate-deficient, but not aliphatic} glucosinolate-deficient mutants, show reduced resistance to $P$. infestans

To further investigate the role of tryptophan-derived secondary metabolites in NHR to P. infestans we selected mutants deficient in different parts of the pathway. We tested NHR to $P$. infestans in mutants deficient in indolic glucosinolates and camalexin (cyp79b2/b3), deficient in camalexin (pad3), reduced in 4MO-I3M (cyp81f2), deficient in PEN2-dependent hydrolysis of 4MO-I3M (pen2-1), and deficient in PEN2-dependent hydrolysis of $4 \mathrm{MO}-\mathrm{I} 3 \mathrm{M}$ and camalexin (pen2-1 pad3) (Fig. 3). cyp79b2/b3, pen2-1, and pen2-1 pad3 showed cell death in response to $P$. infestans inoculation, with 

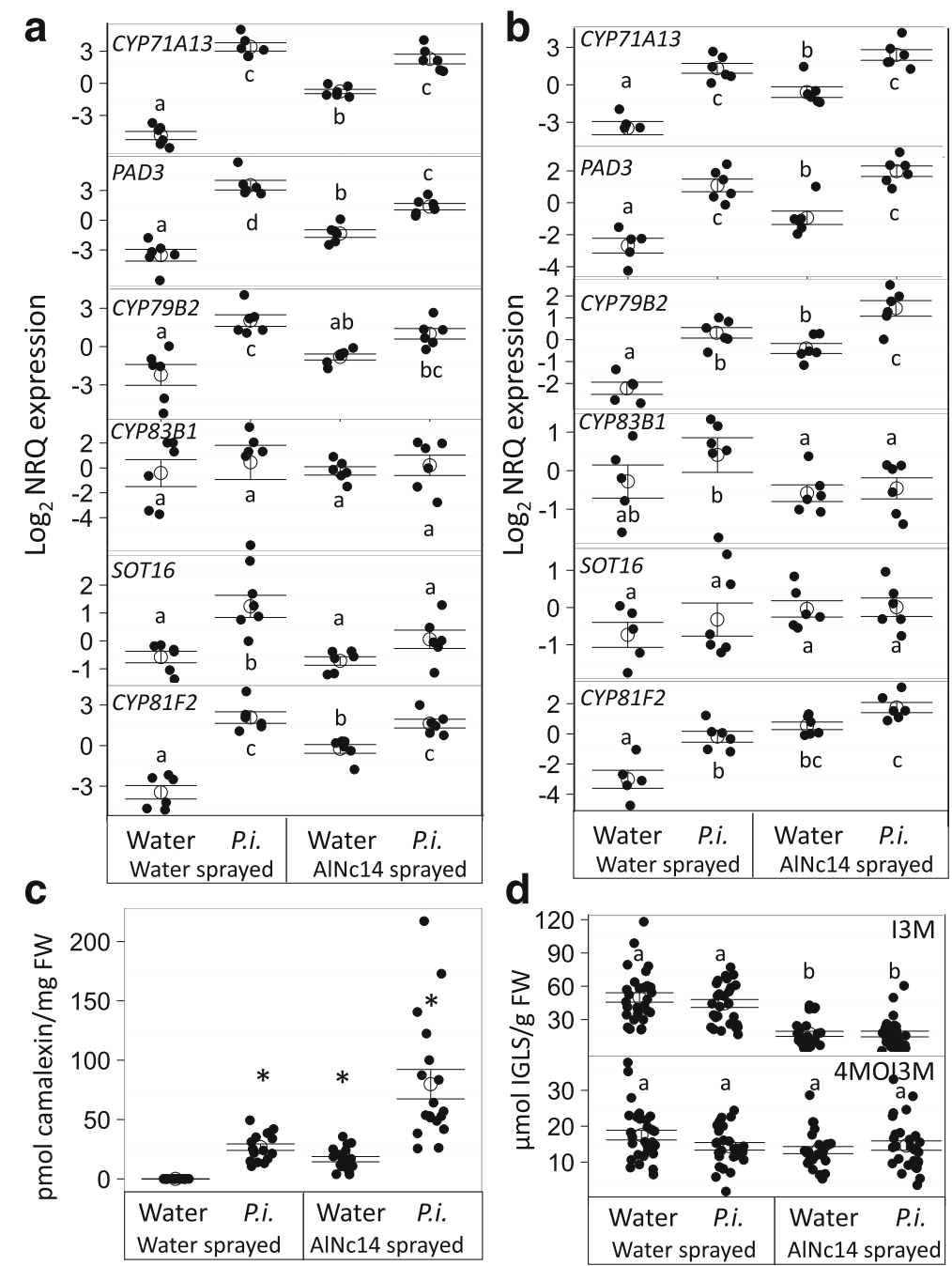

Fig. 4 Albugo infection changes the proportions of camalexin and indolic glucosinolates but does not eliminate them. a and $\mathbf{b}$ Albugo infection changes expression of selected genes in the tryptophan-derived metabolite pathway upon $P$. infestans infection. Open circles and bars denote the mean \pm SE of target gene expression ( $\log _{2}$ transformed normalized relative quantities) in water-sprayed or AlNc14-infected tissue after water or P. infestans $\left(100 \mu \mathrm{L}\right.$ of $1.25 \times 10^{5}$ spores per $\mathrm{mL}$ ) inoculation. a 10 days post inoculation (dpi) with water or AlNc14, 6 hours post inoculation (hpi) with water or $P$. infestans. b 12 dpi with water or AINc14, 48 hpi with water or $P$. infestans. Data are three independent biological replicates with two technical replicates each. Closed, black circles denote individual data points. Different letters indicate significant differences $(P<0.05)$ (Two-way ANOVA with Tukey's honest significance difference). $\mathbf{c}$ Albugo and $P$. infestans infection triggers camalexin accumulation. High-performance liquid chromatography (HPLC) analysis of water-sprayed or AlNc14-infected Col-0 tissue (12 dpi), 48 hours post water or $P$. infestans inoculation ( $100 \mu \mathrm{L}$ of $2.75 \times 10^{5}$ spores per $\mathrm{mL}$ ). Open circles and bars denote the mean camalexin content per $\mathrm{mg}$ fresh weight $\pm \mathrm{SE}$ of three independent biological replicates with six technical replicates each. Closed, black circles denote individual data points. Asterisks indicate significant differences from mock-treated plants (12 dpi water, 48 hpi water). Generalized linear model (GLM) with ${ }^{*} P<0.001$. d Albugo infection decreases I3M levels but does not affect 4MO-I3M levels. HPLC analysis of mock or AINc14-infected Col-0 tissue (12 dpi), 20 hpi mock or P. infestans (100 $\mu \mathrm{L}$ of $3 \times 10^{5}$ spores per $\mathrm{mL}$ ). Open circles and bars denote the mean indolic glucosinolate content per $\mathrm{g}$ of fresh weight \pm SE of five independent biological replicates with six technical replicates each. Closed, black circles denote individual data points. GLM with different letters indicating significant differences $(P<0.001)$

the strongest phenotype observed with cyp79b2/b3 (Fig. 5b, h and i). These observations were complemented by fluorescence microscopy, which revealed that cyp79b2/b3, cyp81f2, pen2-1, and pen2-1 pad3 allowed $P$. infestans growth within the leaf that was visible from the adaxial surface (Fig. 5e, f, k and l). P. infestans was observed to form haustoria (Additional file 14) and occasionally sporulate (between 0 and $8.9 \%$ of leaves; Additional file 15, Fig. 5e) during infection of cyp79b2/ $b 3$ tissue. We quantified the relative amount of $P$. infestans biomass on each mutant compared to Col-0 using qRT-PCR. In agreement with microscopy, $P$. infestans biomass was significantly higher on cyp $79 b 2 / b 3$ than Col-0 or the other mutants $(P<0.05$, Fig. 6a, Additional 


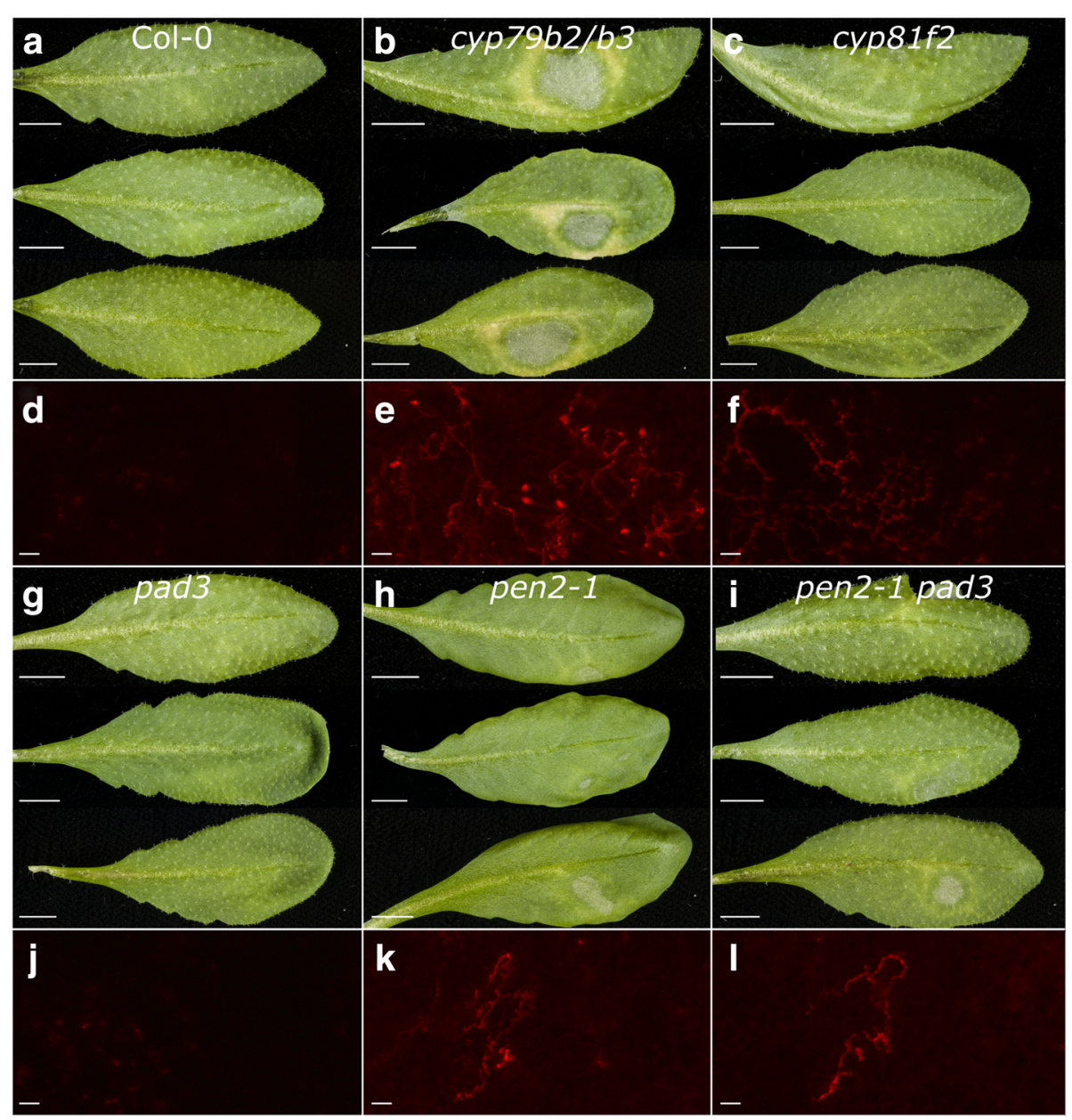

Fig. 5 P. infestans successfully colonizes cyp79b2/b3. P. infestans colonization of mutants in the tryptophan-derived metabolite pathway. a-c, g-i Leaves were inoculated with $100 \mu \mathrm{L}$ of $1 \times 10^{5}$ spores per $\mathrm{mL}$ P. infestans 88069td and photographed at $3 \mathrm{dpi}$. Scale bar: $5 \mathrm{~mm}$. Leaves from three independent experiments are shown. $\mathbf{d}-\mathbf{f}$, j-I Adaxial surface of the leaves was examined using fluorescence microscopy at 3 dpi. Scale bar: 100 $\mu \mathrm{m}$. Three independent experiments were conducted, microscopy from one of the experiments is shown

file 16). We also tested the susceptibility to $P$. infestans of an Arabidopsis line that overproduces brassinosteroid and was reported to have a similar I3M and 4MO-I3M profile to Albugo-infected plants (35S:DWF4 (DWARF4) [89]). 35S:DWF4 was not compromised in NHR to $P$. infestans (Additional files 16). Surprisingly, P. infestans grew less well on 35S:DWF4 plants infected with AlNc14 than on Col-0 plants infected with AlNc14 (Additional file 13 and 16).

Having identified cyp79b2/b3 as compromised in NHR to $P$. infestans we then investigated whether cyp $79 b 2 / b 3$ acts in the same pathway as Albugo in Arabidopsis NHR to $P$. infestans. We infected water- and AlNc14-sprayed Col-0 and cyp79b2/b3 Arabidopsis with P. infestans and quantified $P$. infestans biomass with qRT-PCR. Albugo-infected Col-0 and Albugo-infected cyp $79 b 2 / b 3$ had the same degree of $P$. infestans colonization, which was significantly higher than water-sprayed cyp $79 b 2 / b 3$, which in turn was significantly higher than water-sprayed Col-0 (pre-treatment: $\mathrm{F}_{(1}$, 30) $=270.1, P<0.001$, genotype: $F_{(1,30)}=18.36, P<0.001$, interaction: $\mathrm{F}_{(1,30)}=5.347, P=0.028$; two-way ANOVA with Tukey's HSD) (Fig. 6b, Additional file 17). Albugo-infected Col-0 and Albugo-infected cyp79b2/b3 were more susceptible to $P$. infestans than water-sprayed $c y p 79 b 2 / b 3$, suggesting that deficiency in tryptophan-derived metabolites does not solely explain Albugo-immunosuppression.

To further investigate the role of glucosinolates in $P$. infestans NHR we tested whether aliphatic glucosinolates, which are not derived from tryptophan, play a role. We infected the myb28/29 double mutant, which does not accumulate aliphatic glucosinolates [90], with $P$. infestans. myb28/29 did not allow colonization by $P$. infestans (Additional file 18). We also tested thioglucoside glucohydrolase (tgg)1/tgg2, a mutant in two myrosinases expressed in aerial tissue [91]. $P$. 


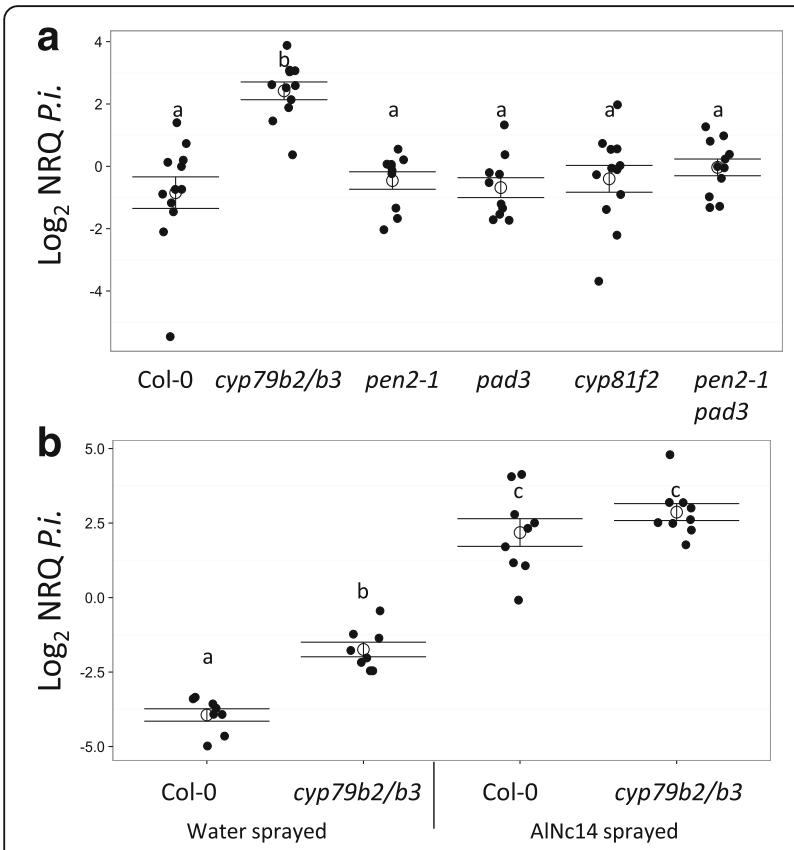

Fig. 6 P. infestans shows increased biomass on cyp7962/b3 compared to Col-0 in the absence of Albugo, but not in its presence. a $P$. infestans biomass on mutants in the tryptophan-derived metabolite pathway. Leaves were inoculated with $100 \mu \mathrm{L}$ of $1 \times 10^{5}$ spores per $\mathrm{mL} P$. infestans 88069td. DNA was extracted at 3 dpi and the proportion of $P$. infestans DNA to plant DNA determined using qRT-PCR. Open circles and bars denote the mean \pm SE of $P$. infestans DNA ( $\log _{2}$ transformed normalized relative quantities (NRQs)) in Arabidopsis tissue from four independent biological replicates with three technical replicates per biological replicate. Closed, black circles denote the individual data points. Different letters indicate significant differences $(P<0.01)$ (Kruskal-Wallis rank sum test with Dunn multiple comparisons test and Bonferroni correction). $\mathbf{b}$ Higher $P$. infestans biomass on AlNc14-infected Arabidopsis than on cyp79b2/b3. Leaves were inoculated with $100 \mu \mathrm{L}$ of $1 \times 10^{5}$ spores per $\mathrm{mL}$ P. infestans $88069 \mathrm{td}$. DNA was extracted at $3 \mathrm{dpi}$ and the proportion of $P$. infestans DNA to plant DNA determined using QRT-PCR. Open circles and bars denote the mean \pm SE of $P$. infestans DNA ( $\log _{2}$ transformed NRQs) in Arabidopsis tissue from three independent biological replicates with three technical replicates per biological replicate. Closed, black circles denote the individual data points. Different letters indicate significant differences $(P<0.01)$ (Two-way ANOVA with Tukey's honest significance difference test)

infestans did not colonize $\operatorname{tgg} 1 / \operatorname{tgg} 2$ (Additional file 18). We therefore conclude that aliphatic glucosinolates play a minimal role in $P$. infestans NHR. In summary, Albugo-suppression of $P$. infestans NHR involves tryptophan-derived secondary metabolites. However, given the increase in $P$. infestans colonization between water-sprayed and Albugoinfected cyp79b2/b3, we conclude that additional changes are imposed by Albugo infection, which promotes P. infestans susceptibility.

\section{Albugo-induced camalexin is biologically unavailable to Botrytis cinerea}

Albugo-infected plants accumulated camalexin (Fig. 4c), which is toxic to necrotrophic fungi including Botrytis cinerea [51, 92, 93]. We therefore tested whether Albugo-infected plants had altered susceptibility to $B$. cinerea by measuring the growth of $B$. cinerea wild type strain B05.10 and mutant $\triangle \mathrm{B}$ catrB4 (lacking a detoxifying $\mathrm{ABC}$ exporter) on water-sprayed and Albugo-infected plants. $\triangle \mathrm{BcatrB} 4$ was more susceptible to camalexin and had reduced virulence on Col-0 but not on the camalexin-deficient mutant pad3 [51].

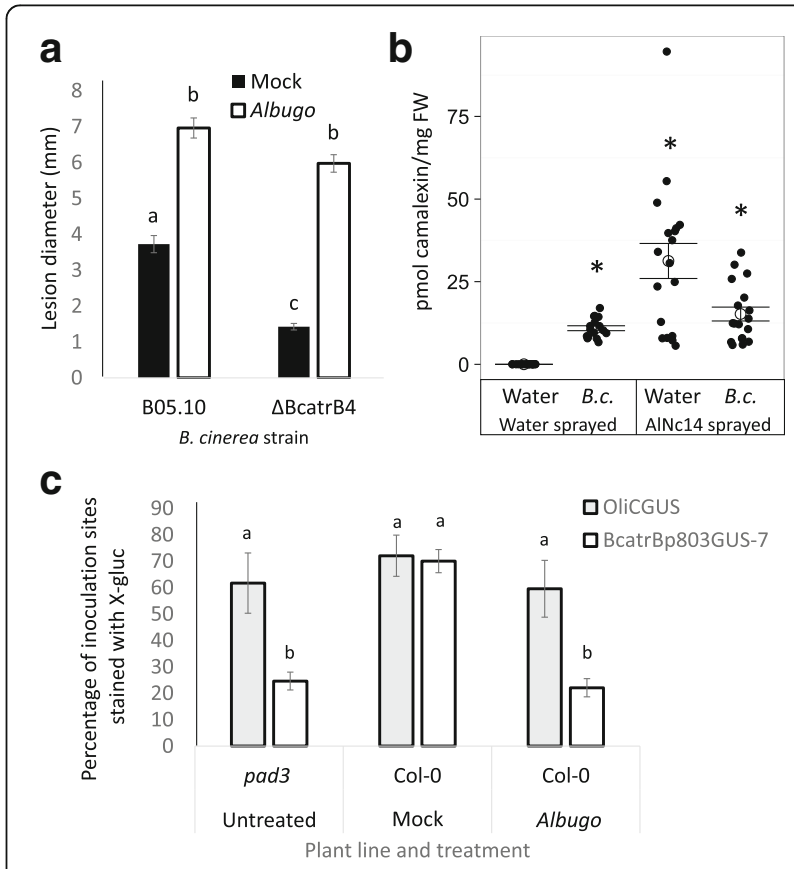

Fig. 7 Albugo-induced camalexin is biologically unavailable to Botrytis cinerea. a $B$. cinerea gives increased disease symptoms on Albugo-infected tissue. Leaves of water-sprayed or AlNc14-infected Col-0 Arabidopsis (11 dpi) were inoculated with $2.5 \times 10^{5}$ spores per $\mathrm{mL}$ of $B$. cinerea B05.10 or camalexin sensitive $\triangle B$ catrB4 mutant, and lesion diameters were measured at 2 dpi. Bars represent mean lesion diameter \pm SE of three independent biological replicates with between 7 and 11 technical replicates per biological replicate $(n=28)$. Different letters indicate significant differences between treatments at $P<0.01$ (Two-way ANOVA with Tukey's honest significance difference). $\mathbf{b}$ Camalexin accumulates in plants infected by Albugo and B. cinerea, either alone or together. High-performance liquid chromatography (HPLC) analysis of mock or AlNc14-infected Col-0 tissue (12 dpi), 26 hours post mock or B. cinerea B05.10 inoculation by spraying $\left(2.5 \times 10^{5}\right.$ spores per $\left.\mathrm{mL}\right)$. Open circles and bars denote mean camalexin content per $\mathrm{mg}$ of fresh weight \pm SE of three independent biological replicates with six technical replicates per biological replicate. Closed, black circles denote individual data points. Asterisks indicate significant differences from mock treated plants (12 days post water spraying, 26 hours post inoculation) at $P<0.001$ (Generalized linear model (GLM)). $\mathbf{c} B$. cinerea detects less available camalexin in Albugo-infected tissue. Leaves of mock or AlNc14-infected Arabidopsis (11 dpi) were drop inoculated with $2.5 \times 10^{5}$ spores per $\mathrm{mL}$ of $B$. cinerea strains OliCGUS (constitutive GUS expression) or BcatBp803GUS-7 (camalexin inducible GUS expression). Leaves were stained with X-gluc at 2 dpi and the percentage of infection sites showing staining determined. Bars represent mean \pm SE three independent biological replicates with between two and four technical replicates per biological replicate (bars left to right $n=5,8,7,10,5,8$ ). Different letters indicate significant differences $P<0.05$ (Three-way ANOVA, Tukey's honest significant difference test) 
We found that B. cinerea B05.10 infection of Albugo-infected plants resulted in lesions almost twice as big as on watersprayed plants (Fig. 7a). The camalexin sensitive $\triangle B$ catrB4 mutant grew significantly less well on water-sprayed plants but produced lesions of a similar size to wild type B05.10 on Albugo-infected plants (Pre-treatment: $\mathrm{F}_{(1,104)}=305.9, P$ $<0.001$, strain: $\mathrm{F}_{(1,104)}=56.31, P<0.001$, interaction: $\mathrm{F}_{(1,}$, ${ }_{104)}=8.713, P<0.01$; two-way ANOVA with Tukey's HSD) (Fig. 7a, Additional file 19). Next, we quantified the accumulation of camalexin in response to B. cinerea B05.10 and AlNc14. Albugo treatment $(\mathrm{z}=-3.409, P<0.001, \mathrm{GLM})$ and B. cinerea inoculation $(\mathrm{z}=9.784, P<0.001)$ led to significant accumulation of camalexin, although the interaction between the two treatments was not significant $(\mathrm{z}=-0.025, P$ $=0.980$ ) (Fig. 7b, Additional file 19). Therefore, the increased susceptibility of Albugo-infected plants to B. cinerea is not due to an overall lack of camalexin accumulation. On the contrary, it suggests that, after Albugo infection, camalexin levels no longer restrict $B$. cinerea proliferation, as lesion sizes are similar in the presence or absence of the detoxifying transporter BcatrB. To assess whether B. cinerea encounters the camalexin present in the plant tissue we used a BcatrB promoter-GUS fusion strain of B. cinerea (BcatrBp803GUS-7). BcatrBp803GUS-7 has low basal expression and is inducible by camalexin [51, 54]. As a control for GUS staining we used the OliCpromoter-GUS fusion $B$. cinerea strain OliCGUS, which shows constitutive expression of the reporter $[53,54]$. We also used pad3 to assess the background expression of BcatrBp803GUS-7 in the absence of camalexin. The two B. cinerea GUS-strains showed similar staining on water-sprayed Col-0 plants but on Albugo-infected Col- 0 plants the GUS expression in BcatrBp803GUS-7 was reduced significantly to levels comparable to when the same strain infected pad 3 plants $(P=$ 0.002) (Pre-treatment: $\mathrm{F}_{(1,37)}=13.449, P<0.001$, strain: $\mathrm{F}_{(1 \text {, }}$ 37) $=19.39, P<0.001$, genotype: $F_{(1,37)}=26.559, P<0.00$, interaction between strain and genotype: $\mathrm{F}_{(1,37)}=13.449, P$ $<0.01$; three-way ANOVA with Tukey's HSD) (Fig. 7c, Additional file 19 and 20). The reduction in GUS production by BcatrBp803GUS-7 on Albugo-infected plants was confirmed by quantifying GUS enzymatic activity using 4-methylumbelliferyl-beta-D-glucuronide (Additional files 13 and 21). These results suggest that, in Albugo-infected plants, $B$. cinerea is exposed to lower camalexin levels than might be expected based on camalexin level measurements in whole leaves.

\section{SA regulated genes during Albugo infection}

As depletion of tryptophan-derived secondary metabolites did not fully mimic the susceptibility of Albugo-infected plants to $P$. infestans we looked for additional candidate pathways in the GO enrichment analysis of the expression profiling. As previously noted, genes upregulated by both pathogens were enriched for GO terms associated with SA signaling (Table 1). To investigate this further, we visualized Arabidopsis genes differentially regulated by the SA mimic BTH [66] in our expression data (Fig. 8a, Additional file 22). The results showed a mixture of responses by BTH-regulated genes to Albugo infection, suggesting a subset of SA responsive genes may be targeted by the pathogens. In particular, a set of genes were less expressed during infection with either pathogen compared to BTH treatment (top of the figure). GO enrichment analysis of Arabidopsis genes differentially expressed specifically by AlNc14 also revealed SA biosynthesis and signaling to be downregulated (Additional file 23).

\section{SA-regulated gene verification}

To confirm the gene expression changes in AlbugoMAGIC 107 interactions mirrored those in Albugo-Col0 interactions we conducted qRT-PCR on AlNc14infected Col-0 Arabidopsis using a set of genes often used as SA markers (PR1, non-inducible immunity1interacting 1 (NIMIN1), WRKY54 and WRKY70 [36, 66, 94, 95]). These genes had different expression profiles over the time course of our data, with PR1 being significantly up-regulated at $4 \mathrm{dpi}$ and not differentially expressed at other time points, WRKY54 being significantly down-regulated at 4,6 , and $8 \mathrm{dpi}$, NIMIN1 being significantly down-regulated at 6 and $8 \mathrm{dpi}$, and WRKY7O being significantly down-regulated at $8 \mathrm{dpi}$ (Additional file 22). Using qRT-PCR we found that, at 10 dpi AlNc14, WRKY54 was significantly down-regulated $(P<0.001)$, while $P R 1$ expression did not significantly change $(P=0.395)$, and WRKY70 and NIMIN1 showed non-significant trends of being down-regulated $(P=$ 0.065 and $P=0.072$, respectively) (Fig. 8b, Additional file 24). These data show similarities to the expression profile data, and therefore suggest that interactions between Albugo and MAGIC 107/Col-0 are likely to be similar.

Recent studies with $\mathrm{Hpa}$ have shown that the pathogen triggers $P R 1$ expression in the surrounding plant tissue while locally suppressing it in haustoriated cells [49, 50]. This cell-specific response is not captured in qRTPCR assays of whole leaves. We used PR1::GUS promoter Arabidopsis line to explore whether AlNc14 suppresses PR1 expression. We combined magenta-GUS staining with trypan blue staining to reveal both the reporter gene induction (purple) and the pathogen (dark blue). In striking contrast to $H p a$, AlNc14 does not trigger high levels of PR1 expression in surrounding tissue (Fig. 8c), suggesting suppression of immunity can be imposed systemically in non-haustoriated cells. We tested whether AlNc14 infection could suppress PR1 induction in response to BTH and SA. Significantly more GUS expression was seen in water-pre-treated plants after BTH and SA treatment compared to AlNc14 pre-treated 

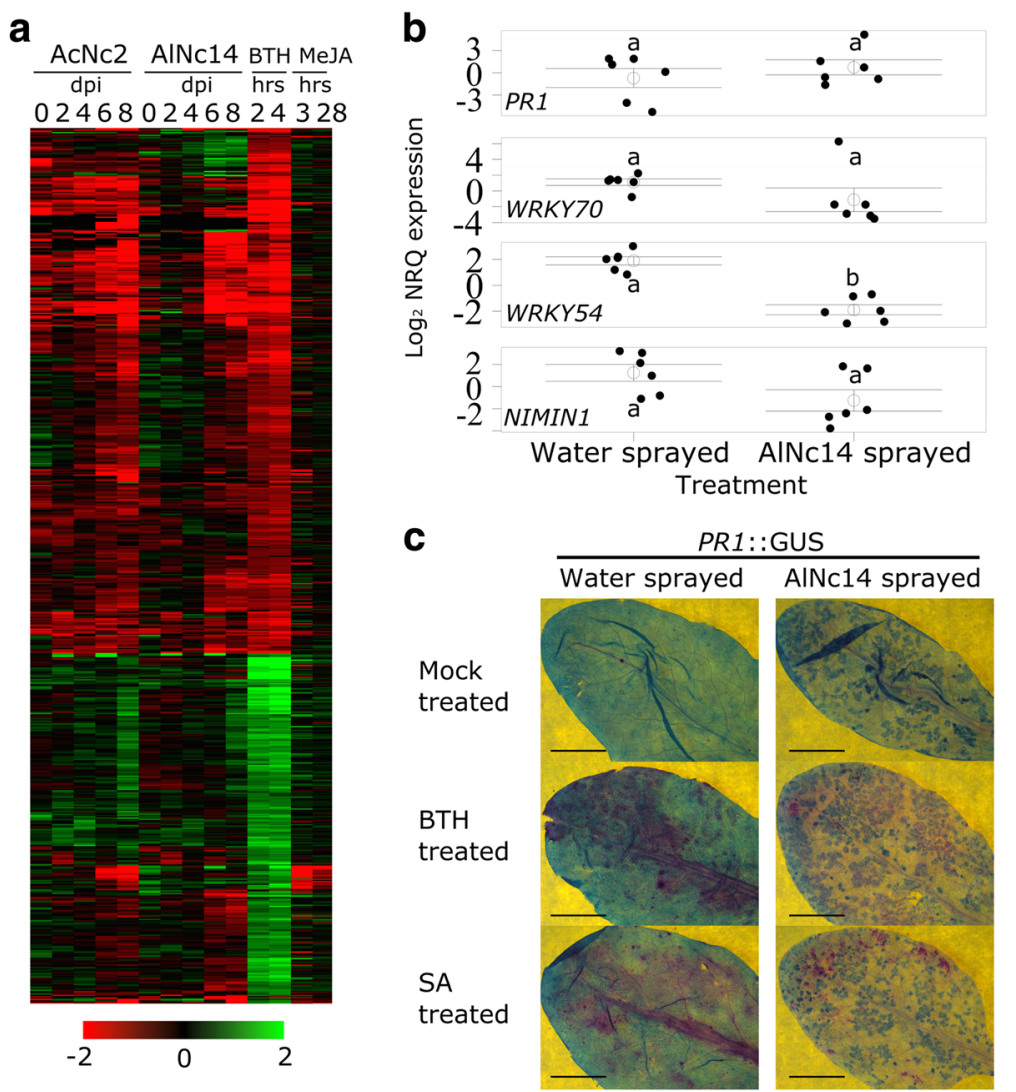

Fig. 8 Albugo-infected leaves reveal reduced expression of salicylic acid (SA)-regulated genes. a Expression pattern of 671 benzo-(1,2,3)-thiadiazole-7carbothioic acid (BTH)-inducible genes reported by [66] after inoculation with AcNc2 and AINc14 over an 8-day time course in MAGIC 107. The data are the average of four experiments. The expression of the same genes during methyl jasmonate treatment $[67,68]$ are shown for comparison. The relative expression (in $\log _{2}$ ratios) is colored red for induction and green for repression as illustrated in the color bar. b Altered SA-regulated gene expression in AINc14 infected Arabidopsis Col-0. Open circles and bars denote the mean \pm SE of target gene expression ( $\log _{2}$ transformed normalized relative quantities) in AlNc14 infected tissue from three independent biological replicates with two technical replicates per biological replicate. Closed, black circles denote the individual data points. Different letters indicate significant differences $(P<0.05)$ in gene expression (Welch Two Sample t-test $(P R 1, P=0.395, W R K Y 54, P<0.001, N I M I N 1$, $P=0.072$ ), Wilcoxon rank sum test (WRKY70, $P=0.065)$ followed by Bonferroni correction). c AINc14 suppresses BTH and SA induction of $P R 1$. To visualize reporter gene induction and pathogen growth in the same leaf, leaves were collected and stained with magenta-GUS to reveal GUS activity, followed by trypan blue to reveal pathogen growth. Leaves of Col-0 pro(PR1):GUS were previously inoculated with water or AlNc14 (13 dpi) and infiltrated with DMSO

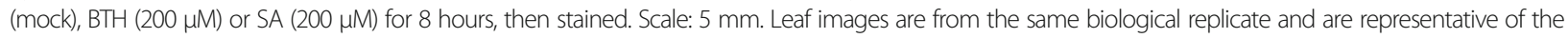
average percentage of staining for each treatment across three independent biological replicates.

plants. The treatments that we compared were inoculation (water or AlNc14: $F_{(1,74)}=21.65, P<0.001$ ), treatment (mock, BTH or SA: $\mathrm{F}_{(1,74)}=84.23, P<0.001$ ), and interaction between inoculation and treatment $\left(\mathrm{F}_{(1,74)}=\right.$ 45.72, $P<0.01$; two-way ANOVA with Tukey's HSD) (Fig. 8c, Additional files 5 and 13). Thus, these data show that AlNc14 can suppress the expression of some of the Arabidopsis genes induced by SA.

\section{SA signaling suppression is not sufficient for susceptibility of Arabidopsis to $P$. infestans}

We next explored whether the suppression of plant SA responses by AlNc14 occurred during the interaction with $P$. infestans, which has been shown to induce $P R 1$ expression at 2-3 dpi in Arabidopsis [16]. To see if AlNc14 suppresses
P. infestans-induced PR1 expression, we infected AlNc14 and water-sprayed PR1::GUS leaves with $P$. infestans. We did not observe the same decrease in magenta GUS staining in the Albugo-inoculated leaves compared to the watersprayed leaves with P. infestans infection (Fig. 9a and b) that was seen for SA and BTH treatments. To further investigate potential suppression of SA responses to $P$. infestans in AlNc14-infected plants, we conducted qRT-PCR on SA marker genes PR1, WRKY 54, and NIMIN1 in leaves of AlNc14-infected or water-sprayed control plants that were subsequently drop inoculated with water or $P$. infestans (Fig. 9c, Additional file 25). PR1 expression did not vary across the treatments (pre-treatment: $F_{(1,19)}=1.066, P=$ 0.315; inoculation: $\mathrm{F}_{(1,19)}=1.075, P=0.313$; interaction: $\mathrm{F}_{(1 \text {, }}$ 19) $=2.428, \quad P=0.136 ; \quad$ two-way ANOVA). WRKY 54 


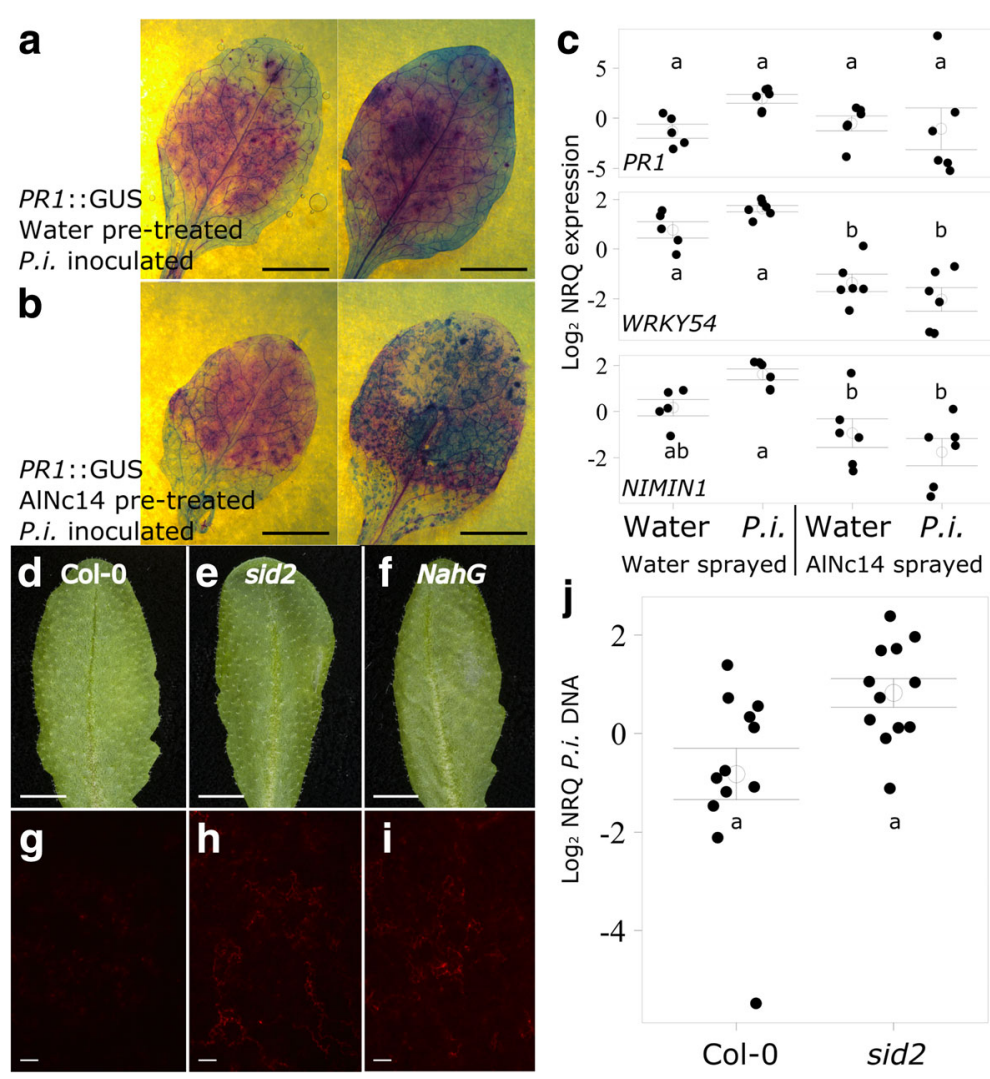

Fig. 9 Albugo suppression of Arabidopsis salicylic acid (SA) responses is not sufficient for full susceptibility to $P$. infestans. a and $\mathbf{b}$ PR1::GUS staining upon $P$. infestans infection. Leaves were collected and stained with magenta-GUS to reveal GUS activity, followed by trypan blue to reveal pathogen growth. PR1::GUS plants were pre-treated with water or AINc14 and subsequently inoculated with $100 \mu \mathrm{L}$ of $1.25 \times 10^{5}$ spores per $\mathrm{mL} P$. infestans 88069td, collected at $2 \mathrm{dpi}$ and stained. Scale: $5 \mathrm{~mm}$. Representative leaves shown are from each of two independent experiments. c AINC14 infection prevents $P$. infestans-induced upregulation of SA marker genes in Col-0. Open circles and bars denote the mean \pm SE of target gene expression ( $\log _{2}$ normalized relative quantities (NRQs)) at 48 hours post treatment $\left(100 \mu \mathrm{L}\right.$ water or $P$. infestans $\left(1.25 \times 10^{5}\right.$ spores per $\left.\mathrm{mL}\right)$ ) of three independent biological replicates with two technical replicates each. Closed, black circles denote the individual data points. Different letters indicate significant differences $(P<0.05$; two-way ANOVA with Tukey's HSD test). $\mathbf{d}-\mathbf{i} P$. infestans partially colonizes sid 2 and NahG Arabidopsis. Leaves were inoculated with $100 \mu \mathrm{L}$ of $1 \times 10^{5}$ spores per $\mathrm{mL}$ P. infestans 88069td, photographed $(\mathbf{d}-\mathbf{f})$ and the adaxial surface examined using fluorescence microscopy $(\mathbf{g}-\mathbf{i})$ at 3 dpi. Red fluorescence denotes $P$. infestans growth, Scale bars: $5 \mathrm{~mm}$ for photographs, $1 \mathrm{~mm}$ for microscopy. Results shown are representative of three independent experiments. $\mathbf{j}$ P. infestans growth on sid2 is not significantly larger than Col-0 Arabidopsis. Leaves were inoculated as in $\mathbf{d}, \mathbf{e}, \mathbf{g}, \mathbf{h}$. DNA was extracted at $3 \mathrm{dpi}$ and the proportion of $P$. infestans DNA to plant DNA determined using qRT-PCR. Open circles and bars denote the mean \pm SE of $P$. infestans DNA ( $\log _{2}$ transformed NRQs) in Arabidopsis tissue from four independent biological replicates with three technical replicates each. Closed, black circles denote the individual data points. The two genotypes were not significantly different $(P=0.012)$ (Wilcoxon rank sum test followed by Bonferroni correction)

expression was significantly decreased in AlNc14infected leaves compared to water-sprayed control leaves (pre-treatment: $F_{(1,19)}=71.520, P<0.001$; inoculation: $F_{(1,19)}=0.026, P=0.8738$; interaction: $F_{(1}$, 19) $=4.796, P=0.041$; two-way ANOVA with Tukey's HSD). NIMIN1 expression was significantly decreased in AlNc14-infected leaves compared to P. infestans inoculated water-sprayed control leaves (pre-treatment: $\mathrm{F}_{(1,19)}=22.096, P<0.001$; inoculation: $\mathrm{F}_{(1,19)}=0.274$, $P=0.607$; interaction: $\mathrm{F}_{(1,19)}=5.327, P=0.032$; twoway ANOVA with Tukey's HSD). In summary, we demonstrated that AlNc14 suppresses P. infestanstriggered NIMIN1 expression and confirmed our previous finding that AlNc14 suppresses WRKY54 expression.

Isochorismate synthase 1 (ics1) (a.k.a. SA-induction deficient 2 (sid2)) is required for SA biosynthesis, and ics1 mutants accumulate very low levels of SA upon pathogen challenge [96]. Since Albugo infection suppresses some of the plant SA responses, we tested whether sid2 was susceptible to $P$. infestans. Observations of infected sid2 leaves showed small amounts of cell death in response to $P$. infestans infection (Fig. 9e). Microscopy revealed a greater degree of tissue colonization in sid2 than Col-0 (Fig. 9g and h), although no $P$. infestans spore formation was observed. A similar phenotype of cell death and increased $P$. infestans 
colonization without spore formation was seen in the NahG Arabidopsis line (Fig. Of and i) which expresses salicylate hydroxylase and degrades SA into catechol [97]. To quantify the amount of $P$. infestans biomass on sid2 compared to Col-0 we estimated relative levels of $P$. infestans DNA using qRT-PCR (Fig. 9j, Additional file 25). Although a trend of increased $P$. infestans colonization of sid 2 was seen $(P=$ 0.012), this was not statistically significant after Bonferroni correction. Taken together, these data suggest that Albugo can suppress a subset of SA responses in Arabidopsis, but the lack of SA responsiveness is unlikely to significantly contribute to the susceptibility of Albugo-infected Arabidopsis to $P$. infestans.

\section{Discussion}

We investigated mechanisms of immuno-suppression by Albugo spp., in particular its remarkable capacity to render Arabidopsis susceptible to the potato late blight pathogen $P$. infestans [12]. Our data reveal alterations in tryptophan-derived secondary metabolite biosynthesis and availability, a role for tryptophan-derived secondary metabolites in Arabidopsis NHR to P. infestans, and suppression of host defense triggered by SA in Albugo-infected tissue.

Confirming that $A$. candida suppresses Arabidopsis NHR to $P$. infestans allowed us to use two Albugo species to investigate shared plant genes altered by Albugo infection through expression profiling. We saw a large number of differentially expressed plant genes between uninfected and infected tissue, which is in contrast to a recent study of the apoplastic proteome of uninfected and A. laibachii-infected tissue that found no significant differences [98]. Surprisingly, the only enriched GO terms in genes downregulated by both pathogens were photosynthesis, commonly downregulated in plants under biotic stress [99], and RNA elongation. The enriched GO terms in genes upregulated by both pathogens were generally related to plant defense responses (SA and JA), again surprising given the immunocompromised nature of the host. Although cells colonized by haustoria may be completely immunosuppressed, adjacent cells may be the source of defense activation revealed in expression profiling, as seen with $\mathrm{Hpa}$ infection [49]. However, we cannot rule out the possibility that Albugo may cause changes in immunity at the protein level in addition to the level of the transcriptome. Changes in secondary metabolites common among Albugo hosts but absent from $P$. infestans hosts can be regarded as plausible candidates for a role in $P$. infestans NHR.

To investigate how Albugo might alter tryptophanderived secondary metabolites, we measured gene expression and metabolite accumulation in response to $P$. infestans in the presence and absence of Albugo. Arabidopsis responds to $P$. infestans inoculation by upregulating the genes involved in camalexin biosynthesis, leading to camalexin accumulation. The main changes in the indolic glucosinolate pathway were an upregulation of SOT16 at early time points and upregulation of CYP81F2 at early and late time points, with no change in the accumulation of $\mathrm{I} 3 \mathrm{M}$ and 4MO-I3M. Accumulation of camalexin and indolic glucosinolates in Arabidopsis in response to non-host pathogens is not uniform. Challenge with biotrophic Bgh leads to no change in camalexin, a decrease in I3M and no change in 4MO-I3M [18], whereas challenge with the necrotrophic fungus Plectosphaerella cucumerina and an incompatible strain of $P$. brassicae leads to an increase in camalexin, a decrease in I3M, and an increase in 4MO-I3M $[32,100]$. Responses to P. infestans in Albugo-infected Arabidopsis were similar to those in plants without Albugo, with the main difference being no significant SOT16 expression and a significant reduction in I3M. The inability to separate I3M from other indole-3-acetaldoxime-derived indolic compounds makes it difficult to test with Arabidopsis mutants whether a reduction in I3M but not camalexin contributes to $P$. infestans NHR. CYP83B1 mutants accumulate increased indole-3-acetic acid, resulting in pleiotropic effects (e.g., [101, 102]), whereas SOT16 mutants are yet to be characterized but may also have a similar phenotype. 35S:DWF4 has reduced I3M compared to Col-0 and similar amounts of 4MO-I3M [89], but we found that this plant line was not susceptible to $P$. infestans in the absence of Albugo and was less susceptible than Col-0 in the presence of Albugo. While the transcriptional responses to $P$. infestans were similar in uninfected and Albugo-infected tissue, the response per amount of $P$. infestans was much lower in the Albugo-infected tissue due to increased $P$. infestans colonization in this tissue.

cyp $79 b 2 / b 3$ is deficient in tryptophan-derived secondary metabolites including indolic glucosinolates and camalexin $[103,104]$ and is the first Arabidopsis mutant, to our knowledge, on which $P$. infestans can sporulate, if only occasionally. As the pen $2-1$ pad3 mutant, deficient in camalexin and hydrolysis of $4 \mathrm{MO}-\mathrm{I} 3 \mathrm{M}$, did not show the same level of $P$. infestans colonization as cyp79b2/ $b 3$, we conclude that tryptophan-derived antimicrobial metabolites, in addition to camalexin and indolic glucosinolates, play a role in P. infestans NHR in Arabidopsis. Our data agree with recent reports [32, 100, 105] of uncharacterized tryptophan-derived secondary metabolites that play an important role in immunity to nonadapted filamentous pathogens. The recent discovery that Arabidopsis synthesizes 4-hydroxyindole-3-carbonyl nitrile from tryptophan, and that mutants in its biosynthesis are more susceptible to the hemibiotroph bacterial pathogen Pseudomonas syringae [106], emphasizes that other molecules contributing to plant defense may remain to be discovered.

Albugo-infected cyp79b2/b3 mutants support more $P$. infestans growth than uninfected cyp $79 b 2 / b 3$, suggesting 
that either Albugo-infection has a stronger phenotype than the cyp $79 b 2 / b 3$ mutant, or mechanisms in addition to indole glucosinolates, camalexin, and tryptophanderived metabolites contribute to $P$. infestans resistance, and that these mechanisms are also suppressed by Albugo infection. The Albugo-infected mutant was not more susceptible than infected Col-0, suggesting that indole-derived metabolites are less effective at suppressing microbial growth in Albugo-infected plant tissue. If Albugo suppression of NHR was working separately to tryptophan-derived secondary metabolites, then we would expect that Albugo-infected plants of cyp $79 b 2 / b 3$ would show additional enhanced susceptibility compared to Albugo-infected Col-0. This suggests that there is interplay between NHR and tryptophan-derived secondary metabolites, although conceivably the additive phenotype was overlooked due to technical limitations. In addition to tryptophan-derived secondary metabolites, we also identified a very minor role for SATI in Arabidopsis NHR to P. infestans, but it is possible that other aspects of plant immunity contribute too.

Albugo-infected plants accumulate camalexin in the absence and presence of $B$. cinerea. However, both wild type $B$. cinerea and the camalexin-sensitive mutant $\triangle B$ catrB4 produce bigger lesions on Albugo-infected plants, while the BcatrBp803GUS-7 B. cinerea strain responds as if the amount of camalexin in Albugo-infected plants is the same as in a camalexin-deficient pad3 mutant. We therefore conclude that the camalexin must be biologically unavailable to $B$. cinerea, and also possibly to $P$. infestans. How camalexin is made biologically unavailable remains to be determined. Conceivably, Albugo infection leads to the compartmentalization of camalexin away from $B$. cinerea and other pathogens potentially accumulated within the Albugo cells. Alternatively, camalexin may be modified by Albugo in some way to make it biologically inert, though no such modification is visible in our metabolomics analysis. A recent study demonstrated that metabolites inhibiting the germination of $P$. infestans spores required secreting to the leaf surface to be effective [107]; therefore, it is also possible that Albugo alters metabolite transport, and hence spatial distribution. Whether altering tryptophan-derived metabolite biosynthesis and availability provides an advantage to Albugo, and is a direct result of Albugo effectors, remains unresolved. Some pathogens, such as the maize smut fungus Ustilago maydis, use effectors to manipulate plant metabolism to their advantage $[108,109]$. Other pathogens have been shown to detoxify plant phytoalexins by active transport [51] or enzymatic modification [33-35]. Tryptophan-derived secondary metabolites are unlikely to be essential for Albugo infection of Arabidopsis, as Albugo can infect cyp79b2/b3 and reduce NHR to $P$. infestans to the same extent as Col- 0 .

We also investigated SA-responsive gene expression in Albugo-infected tissue. We conducted qRT-PCR to investigate the expression of four SA marker genes identified in the expression profiling. The qRT-PCR largely matched the expression profiling, with WRKY54 being significantly down-regulated, WRKY70 and NIMIN1 showing less expression, and $P R 1$ showing no change. We also used PR1::GUS reporter lines and SA/BTH to show that Albugo suppresses $P R 1::$ GUS transcription in the presence of SA/ BTH. The suppression of SATI by Albugo provides a potential explanation for the observation that A. laibachii colonization is not significantly increased on sid2 compared with Col-0 [98], and may also partly explain the impairment of host resistance against other pathogens [10, 11]. We have proposed that defense suppression is not only necessary for the pathogen's own colonization, but also may allow different isolates to co-exist on a common host in order to facilitate hybridization between races that would not otherwise colonize the same host [10].

$P$. infestans induces expression of PR1::GUS in Arabidopsis [16]. Albugo-infected Arabidopsis does not show the clear suppression of PR1::GUS expression upon $P$. infestans challenge that was seen with BTH and SA. SA marker gene expression was not significantly induced in our qRT-PCR experiments with $P$. infestans. This may be because expression is localized to the site of inoculation, therefore being diluted at the whole leaf level, or the level of expression induced by $P$. infestans is relatively small. Alternatively, a more frequent time course experiment could be conducted to identify whether these genes peak in expression. NIMIN1 was significantly down-regulated upon $P$. infestans challenge in Albugo-infected tissue compared to uninfected tissue, thus providing evidence that SATI to $P$. infestans is compromised in the presence of Albugo. Arabidopsis mutants in SATI are more susceptible to P. capsici [31]. A slight decrease in resistance, e.g., trailing necrosis, was also observed upon infection of NahG and nonexpresser of $\mathrm{pr}$ genes 1 (npr1) plants after inoculation with an incompatible strain of $P$. brassicae [110]. The SA biosynthesis mutant sid2 supported more $P$. infestans colonization compared to Col-0. Our results differ from a recent report of $P$. infestans infection of $s i d 2$, which did not identify any increase in $P$. infestans colonization or any increased cell death compared to Col-0 [25]. This may be due to a difference in the $P$. infestans strains used or the conditions for the experiments. We did not observe $P$. infestans spore formation on sid 2 Arabidopsis, unlike Albugo-infected tissue and cyp79b2/b3. This suggests that the contribution of SATI to $P$. infestans NHR is likely to be minor.

\section{Conclusions}

Previously, Albugo suppression of plant immunity had been described but the mechanisms involved had not been investigated. Now, the identification of Albugo-induced alterations in tryptophan-derived secondary metabolite biosynthesis and availability and suppression of SATI will inform more 
focused studies on potential Albugo effectors, as for other pathogens and pests $[111,112]$, by providing phenotypes to screen for. Identification of proteins that are recognized by plants, leading to resistance against Albugo will also help identify likely effectors. In the future, it may be possible to take advantage of the apparent conservation of function of secondary metabolites in plant immunity [27] by using tryptophan-derived secondary metabolites and other phylogenetically limited metabolites in crop protection strategies against $P$. infestans and other pathogens or pests, either through direct application of the metabolites or by transgenically transferring the metabolic pathways into new species.

\section{Additional files}

Additional file 1: Plant lines used in the study. A list of the Arabidopsis ecotypes, crossed lines, mutants and transgenic lines used in the study. All mutants and transgenic lines are in the Col-0 background except pen2-1 and Col-gl RPW8.1 RPW8.2, which are in the glabrous 7 background. (DOCX $18 \mathrm{~kb}$ )

Additional file 2: Arabidopsis genes differently regulated in expression profiling through randomly sheared CDNA tag sequencing (EXPRSS) data. Lists of the Arabidopsis genes that were differentially expressed in AINC2 and AINc14 infected tissue over a time course. (XLSX $1019 \mathrm{~kb}$ )

Additional file 3: Differentially regulated genes at each time point and overlap between genes regulated by each pathogen. Comparisons of the Arabidopsis genes differentially expressed during infection with AINc2 and/or AlNc14 over a time course. (XLSX $104 \mathrm{~kb}$ )

Additional file 4: Primers used in the study. Details of the primers used to conduct qRT-PCR in the study. (DOCX $25 \mathrm{~kb}$ )

Additional file 5: AINC14 suppresses benzo-(1,2,3)-thiadiazole-7carbothioic acid (BTH) and salicylic acid (SA) induction of PR1. To visualize reporter gene induction and pathogen growth in the same leaf, leaves were collected and stained with magenta-GUS to reveal GUS activity, followed by trypan blue to reveal pathogen growth. Leaves of Col-0 pro(PR1)::GUS were previously inoculated with water or AINc14 (13 dpi)

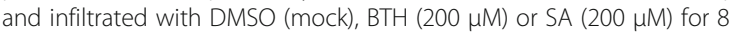
hours, then stained and examined with a microscope. The percentage of each leaf stained with GUS was determined using ImageJ. (A) Open circles represent mean \pm SE of the raw data (percentage of leaf stained) of three independent biological replicates with between two and seven technical replicates per biological replicate (bars left to right $n=10,12,13$, 15,14 and 16). (B) Open circles represent mean \pm SE of the transformed data (arcsine square root transformation followed by $\log _{10}$ transformation) of three independent biological replicates with between two and seven technical replicates per biological replicate (bars left to right $n=10,12,13$, 15,14 and 16). Different letters indicate significant differences $P<0.001$ (Two-way ANOVA, Tukey's HSD test). (PDF 33 kb)

Additional file 6: Hyaloperonospora arabidopsidis (Hpa) Waco9 infection does not allow $P$. infestans colonization of Arabidopsis. (A) Water sprayed, (B) AlNc14 sprayed (12 dpi) and (C) Hpa sprayed leaves (6 dpi) were drop inoculated with $100 \mu \mathrm{L}$ of 3.25 to $5 \times 10^{4}$ spores per $\mathrm{mL} P$. infestans 88069 td. Fluorescence microscopy of the adaxial surface of the leaf taken at $3 \mathrm{dpi}$ P. infestans. Scale bar: $100 \mu \mathrm{m}$. Results shown are representative of two independent experiments. (TIF $3605 \mathrm{~kb}$ )

Additional file 7: Gene ontology (GO) terms within biological processes that are significantly enriched amongst genes up-regulated in the expression profiling. Results of the $\mathrm{GO}$ enrichment analysis for up-regulated genes. (XLSX $328 \mathrm{~kb})$

Additional file 8: Gene ontology (GO) terms within biological processes that are significantly enriched amongst genes down-regulated in the expression profiling. Results of the GO enrichment analysis for downregulated genes. (XLSX $157 \mathrm{~kb}$ )
Additional file 9: Two-way ANOVA results from qRT-PCR of tryptophanderived secondary metabolite genes at 6 hours post $P$. infestans inoculation. ANOVA table. (DOCX $13 \mathrm{~kb}$ )

Additional file 10: Data analyzed in Fig. 4. Spreadsheets showing the data analyzed in Fig. 4a-d. (XLSX $30 \mathrm{~kb}$ )

Additional file 11: Two-way ANOVA results from qRT-PCR of tryptophanderived secondary metabolite genes at 48 hours post $P$. infestans inoculation. ANOVA table. (DOCX $13 \mathrm{~kb}$ )

Additional file 12: Indolic glucosinolate measurements in pen2-1 plants in response to pre-treatment with water or Albugo and subsequent inoculation with water or P. infestans. HPLC analysis of mock or Albugo infected pen2-1 tissue (12 dpi), 20 hours post mock or $P$. infestans treatment (100 $\mu \mathrm{L}$ of $3 \times 10^{5}$ spores per $\mathrm{mL}$ ). Open circles and bars denote mean indolic glucosinolate content \pm SE of three independent biological replicates with six technical replicate per biological replicate. Closed, black circles denote the individual data points. Different letters indicate significant different values within each glucosinolate measured $(P<0.05)$ (Two-way ANOVA, Tukey's HSD test). (PDF $175 \mathrm{~kb}$ )

Additional file 13: Data analyzed in Additional files. Spreadsheets showing the data analyzed in Additional files 11, 16 and 20. (XLSX $27 \mathrm{~kb}$ )

Additional file 14: $P$. infestans forms haustoria in cyp79b2/b3 plants. Leaves of Nicotiana benthamiana (A and B) and Arabidopsis cyp7962/b3 (C and D) were drop inoculated with $50 \mu \mathrm{L}$ of $1 \times 10^{5}$ spores per $\mathrm{mL} P$. infestans $88069 \mathrm{td}$ and examined using confocal microscopy at $2 \mathrm{dpi}$ ( $\mathrm{A}$ and $\mathrm{B}$ ) and $3 \mathrm{dpi}$ (C and D). A and C show colonization of the leaf by P. infestans. Scale bar $=100 \mu \mathrm{M}$. B and $D$ show formation of infection structures by $P$. infestans, with haustoria denoted by asterisks. Scale bar $=10 \mu \mathrm{M}$. (PDF $485 \mathrm{~kb}$ )

Additional file 15: $P$. infestans sporulation on cyp79b2/b3 plants. Leaves of Col-0 and cyp79b2/b3 were inoculated with $100 \mu \mathrm{L}$ of $2.5 \times 10^{5}$ spores per $\mathrm{mL}$ P. infestans NL12226. Photographs were taken of the abaxial surface of Col-0 (A) and cyp79b2/b3 (B) leaves at $3 \mathrm{dpi}$. Scale bars: $5 \mathrm{~mm}$. ( $C$ and D) Leaves were examined for sporulation between 3 and 5 dpi by placing water droplets on the leaves and examining them for the presence of spores using a light microscope (C). Frequency of sporulating leaves in three independent experiments were recorded (D) (Replicate 1: Col-0, $\mathrm{n}=44$, sporulating $=0 ;$ cyp79b2/b3, $\mathrm{n}=56$, sporulating $=5$. Replicate $2:$ Col-0 $\mathrm{n}=42$, sporulating $=0 ;$ cyp7962/b3, $\mathrm{n}=62$, sporulating $=4$. Replicate $3:$ Col- 0 , $\mathrm{n}=22$, sporulating $=0 ; c y p 7962 / b 3, \mathrm{n}=71$, sporulating $=0$ ). (TIF $9710 \mathrm{~kb}$ )

Additional file 16: Albugo-infected 35S:DWF4 is less susceptible to $P$. infestans than Albugo-infected Col-O. (A-D) Fluorescence microscopy of the adaxial surface of water-sprayed Col-0 (A), water-sprayed 35S:DWF4 (B), Albugoinfected Col-0 (C) and Albugo-infected 35S:DWF4 (D) leaves. Leaves were sprayed with water or Albugo and subsequently inoculated (12 days post spraying) with $100 \mu \mathrm{L}$ of $1 \times 10^{5}$ spores per $\mathrm{mL} P$. infestans 88069td. Leaves were examined using fluorescence microscopy at $3 \mathrm{dpi}$. Red fluorescence denotes P.i. growth. Scale bars: $200 \mu \mathrm{m}$. Results shown are representative of three independent experiments. (E and F) Photographs of Albugo-infected Col-0 (E) and Albugo-infected 35S:DWF4 (F) leaves, infected as described above, were taken at 3 dpi. Scale bars: $5 \mathrm{~mm}$. (G) Quantification of P. infestans biomass on Albugo infected Col-0 and 35S:DWF4 by qRT-PCR. Leaves were inoculated with $100 \mu \mathrm{L}$ of $1 \times 10^{5}$ spores per $\mathrm{mL} P$. infestans $88069 \mathrm{td}$. DNA was extracted at 3 $\mathrm{dpi}$ and the proportion of $P$. infestans DNA to plant DNA determined using qRT$P C R$. Open circles and bars denote means \pm SE of three independent biological replicates with three technical replicates per biological replicate. Closed, black circles denote the individual data points. Different letters indicate significant differences (Welch two sample t-test) $(P<0.001$ ). (TIF $3282 \mathrm{~kb}$ )

Additional file 17: Data analyzed in Fig. 6. Spreadsheets showing the data analyzed in Fig. $6 a$ and b. (XLSX 17 kb)

Additional file 18: $m y b 28 / 29$ and $\operatorname{tg} 1 / \operatorname{tgg} 2$ are not susceptible to $P$. infestans. (A-C) Leaves of Col-0, myb28/29 and cyp79b2/b3 (positive control) were inoculated with $100 \mu \mathrm{L}$ of at least $1 \times 10^{5}$ spores per $\mathrm{mL} P$. infestans 88069td. (D-F) Leaves of Col-0, tgg 1/2 and AlNc14 sprayed Col-0 (positive control) were inoculated with $100 \mu \mathrm{L}$ of at least $1 \times 10^{5}$ spores per $\mathrm{mL}$ P. infestans 88069td. The adaxial surface of the leaves was examined using fluorescence microscopy at 3 dpi. Scale bars: $100 \mu \mathrm{m}$. Red fluorescence denotes P.I. growth. Results shown are representative of two (A-C) and three (D-F) independent experiments. (TIF $5249 \mathrm{~kb}$ ) 
Additional file 19: Data analyzed in Fig. 7. Spreadsheets showing the data analyzed in Fig. 7a-c. (XLSX 17 kb)

Additional file 20: Example of staining of GUS-expressing B. cinerea strains on water and Albugo-sprayed Col-0 leaves. Photograph of three representative leaves. Top row are AINc14-infected leaves and the bottom row are water-sprayed leaves. The left hand side of each leaf received three droplets of OliCGUS $B$. cinerea and the right hand side received three drops of BcatrBp803GUS-7 B. cinerea. Leaves were removed from the plant and stained at $2 \mathrm{dpi} B$. cinerea. (TIF $6351 \mathrm{~kb}$ )

Additional file 21: $B$. cinerea detects less available camalexin in Albugoinfected tissue. Leaves underwent protein extraction and GUS enzyme activity was determined using a fluorescence-based assay. Results were normalized to $B$. cinerea weight proportion of each sample using qRTPCR on Botrytis and Arabidopsis genomic DNA. Open circles and bar dots represent the mean \pm SE of three independent biological replicates with three or four technical replicates per biological replicate. Closed, black circles denote the individual data points. Asterisk indicates significant differences measured at $P<0.05$ (Wilcoxon rank sum test within $B$. cinerea strain followed by Bonferroni correction), n.s. = not significant. (PDF $176 \mathrm{~kb}$ )

Additional file 22: Benzo-(1,2,3)-thiadiazole-7-carbothioic acid (BTH) regulated genes during Albugo infection time course. Spreadsheet showing the expression of Arabidopsis BTH regulated genes during infection with Albugo. (XLSX $346 \mathrm{~kb}$ )

Additional file 23: List of selected, lower level gene ontology (GO) terms enriched in genes differentially expressed during AlNc14 infection but not AcNc2 infection. Table showing the GO terms enriched in Arabidopsis genes differentially expressed during AlNc14 infection only. (DOCX $14 \mathrm{~kb}$ )

Additional file 24: Data analyzed in Fig. 8. Spreadsheets showing the data analyzed in Fig. 8b. (XLSX 14 kb)

Additional file 25: Data analyzed in Fig. 9. Spreadsheets showing the data analyzed in Fig. $9 \mathrm{c}$ and j. (XLSX $16 \mathrm{~kb}$ )

\section{Abbreviations}

4MO-I3M: 4-methoxyindol-3-ylmethylglucosinolate; ABC: ATP-binding cassette Ac: Albugo candida; ANOVA: analysis of variance; BTH: benzo- $(1,2,3)$-thiadiazole7-carbothioic acid; CER: controlled environment room; CYP: cytochrome P450; dpi: days post inoculation; DWF4: dwarf 4; EDTA: ethylenediaminetetraacetic acid; ETI: effector-triggered immunity; EXPRSS: expression profiling through randomly sheared CDNA tag sequencing; FDR: false discovery rate; GLM: generalized linear model; GO: gene ontology; GUS: $\beta$-glucuronidase; Hpa: hyaloperonospora arabidopsidis; hpi: hours post inoculation; HPLC: high performance liquid chromatography; HSD: honest significant difference; I3M: indol-3-ylmethylglucosinolate; JA: jasmonic acid; MAGIC: multiparent advanced generation inter-cross; NHR: non-host resistance; NIMIN1: noninducible immunity1-interacting 1; NRQs: normalized relative quantities; PAD3: phytoalexin deficient 3; PEN: penetration; PR1: pathogenesis-related 1; PTI: pattern-triggered immunity; SA: salicylic acid; SATI: SA-triggered immunity; SOT16: sulfotransferase 16; TAIR10: The Arabidopsis Information Resource version 10

\section{Acknowledgements}

We thank Jan van Kan (Laboratory of Phytopathology, Wageningen University and Research, Wageningen) for providing B. cinerea wildtype strain B05.10, Michaela Leroch (University of Kaiserslautern, Kaiserslautern) for B. cinerea strains BcatrBp803GUS and OliCGUS, and Geert Kessel (Biotic Interactions and Plant Health, Wageningen University and Research, Wageningen) for providing P. infestans isolate NL12226. We thank Georg Jander (Boyce Thompson Institute for Plant Research, Ithaca; tgg1 tgg2), Paul Schulze-Lefert (Max Planck Institute for Plant Breeding Research, Cologne; pen2-1 pad3), and Cyril Zipfel (The Sainsbury Laboratory, Norwich; 35S:DWF4) for providing Arabidopsis seeds. We are grateful to Dan MacLean for advice on statistical analysis. We acknowledge Andrew Davies for photographic services and the John Innes Horticultural Services for growing the plants used in the study.

\section{Funding}

The authors thank European Research Council Advanced Investigator grant 233376 (ALBUGON) (to JDGJ), the Gatsby Foundation (http://www.gatsby.org.uk), the UK Biotechnology and Biological Sciences Research Council (BBSRC) grant BB/ G042960/1 as part of the ERA-PG consortium "PRR-CROP" (to HS), Danish National Research Foundation grant number 99 (to BAH), and Japan Society for the Promotion of Science KAKENHI 15 K18651 and a RIKEN Special Postdoctoral Research Fellowship (to SA) for funding. The funders had no role in study design, data collection and analysis, decision to publish, or preparation of the manuscript.

\section{Availability of data and materials}

The datasets supporting the conclusions of this article are either included within the article (and its additional files) or are available from Gene Expression Omnibus under series accession number GSE75016.

\section{Authors' contributions}

Design of the research: DCP and JDGJ. Performance of the research: DCP, GR, DX, HS, VC, SA, EK, NCM, AK, KB. Data analysis/collection/interpretation: DCP, GR, DX, HS, VC, SA, NCM, KB, SS, SK EH, BAH, JDGJ. Writing of the manuscript: DCP and JDGJ. All authors commented on various drafts of the manuscript and read and approved the final manuscript.

\section{Competing interests}

The authors declare that they have no competing interests.

\section{Consent for publication}

Not applicable.

\section{Ethics approval and consent to participate}

Not applicable.

\section{Author details}

${ }^{1}$ The Sainsbury Laboratory, Norwich Research Park, Norwich NR4 7UH, United Kingdom. ${ }^{2}$ School of Biological Sciences, University of East Anglia, Norwich Research Park, Norwich, United Kingdom. ${ }^{3}$ Norwich Medical School, University of East Anglia, Norwich Research Park, Norwich, UK. ${ }^{4}$ DynaMo Center, Department of Plant and Environmental Sciences, Faculty of Science, University of Copenhagen, 40 Thorvaldsensvej, DK-1871 Frederiksberg C, Denmark. ${ }^{5}$ Department of Crop Genetics, John Innes Centre, Norwich Research Park, Norwich NR4 7UH, UK. ${ }^{6}$ Department of Biology and Biochemistry, University of Bath, Bath, UK. ${ }^{7}$ Plant Immunity Research Group, Center for Sustainable Resource Science, RIKEN Yokohama Institute, Yokohama, Japan. ${ }^{8}$ Max Planck Research Group Fungal Biodiversity, Max Planck Institute for Plant Breeding Research, Cologne, Germany. ${ }^{9}$ Sainsbury Laboratory, University of Cambridge, Cambridge, UK. ${ }^{10}$ School of Life Sciences, Warwick Crop Centre, University of Warwick, Wellesbourne, UK.

Received: 16 December 2016 Accepted: 22 February 2017

Published online: 20 March 2017

\section{References}

1. Jones JDG, Dangl JL. The plant immune system. Nature. 2006:444(7117):323-9.

2. Dodds PN, Rathjen JP. Plant immunity: towards an integrated view of plantpathogen interactions. Nat Rev Genet. 2010;11(8):539-48.

3. Dangl JL, Horvath DM, Staskawicz BJ. Pivoting the plant immune system from dissection to deployment. Science. 2013;341(6147):746-51.

4. Stahl EA, Dwyer G, Mauricio R, Kreitman M, Bergelson J. Dynamics of disease resistance polymorphism at the Rpm1 locus of Arabidopsis. Nature. 1999:400(6745):667-71.

5. Heath MC. Nonhost resistance and nonspecific plant defenses. Curr Opin Plant Biol. 2000;3(4):315-9.

6. Mysore KS, Ryu C-M. Nonhost resistance: how much do we know? Trends Plant Sci. 2004;9(2):97-104.

7. Schulze-Lefert P, Panstruga R. A molecular evolutionary concept connecting nonhost resistance, pathogen host range, and pathogen speciation. Trends Plant Sci. 2011;16(3):117-25.

8. Thines M. Phylogeny and evolution of plant pathogenic oomycetes-a global overview. Eur J Plant Pathol. 2014;138(3):431-47.

9. Thines M, Choi YJ, Kemen E, Ploch S, Holub EB, Shin HD, et al. A new species of Albugo parasitic to Arabidopsis thaliana reveals new evolutionary patterns in white blister rusts (Albuginaceae). Persoonia. 2009;22:123-8. 
10. McMullan M, Gardiner A, Bailey K, Kemen E, Ward BJ, Cevik V, et al. Evidence for suppression of immunity as a driver for genomic introgressions and host range expansion in races of Albugo candida, a generalist parasite. elife. 2015;4:e04550.

11. Cooper AJ, Latunde-Dada AO, Woods-Tör A, Lynn J, Lucas JA, Crute IR, et al. Basic compatibility of Albugo candida in Arabidopsis thaliana and Brassica juncea causes broad-spectrum suppression of innate immunity. Mol PlantMicrobe Interact. 2008;21(6):745-56.

12. Belhaj K, Cano LM, Prince DC, Kemen A, Yoshida K, Dagdas YF, et al. Arabidopsis late blight: infection of a nonhost plant by Albugo laibachii enables full colonization by Phytophthora infestans. Cell Microbiol. 2017;19(1):e12628

13. Haverkort AJ, Struik PC, Visser RGF, Jacobsen E. Applied biotechnology to combat late blight in potato caused by Phytophthora infestans. Potato Res. 2009;52(3):249-64.

14. Van Damme M, Schornack S, Cano LM, Huitema E, Kamoun S. Interactions between Phytophthora infestans and Solanum. In: Lamour K, Kamoun S, editors. Oomycete Genetics and Genomics: Diversity, Interactions and Research Tools. Hoboken, New Jersey: Wiley-Blackwell; 2009. p. 287-302.

15. Vleeshouwers VGAA, van Dooijeweert W, Govers F, Kamoun S, Colon LT. The hypersensitive response is associated with host and nonhost resistance to Phytophthora infestans. Planta. 2000;210(6):853-64.

16. Huitema E, Vleeshouwers VGAA, Francis DM, Kamoun S. Active defence responses associated with non-host resistance of Arabidopsis thaliana to the oomycete pathogen Phytophthora infestans. Mol Plant Pathol. 2003;4(6):487-500

17. Westphal L, Scheel D, Rosahl S. The coi1-16 mutant harbors a second site mutation rendering PEN2 nonfunctional. Plant Cell Online. 2008;20(4):824-6.

18. Bednarek P, Piślewska-Bednarek M, Svatoš A, Schneider B, Doubský J, Mansurova $M$, et al. A glucosinolate metabolism pathway in living plant cells mediates broad-spectrum antifungal defense. Science. 2009;323(5910):101-6.

19. Stein M, Dittgen J, Sánchez-Rodríguez C, Hou B-H, Molina A, Schulze-Lefert $P$, et al. Arabidopsis PEN3/PDR8, an ATP binding cassette transporter, contributes to nonhost resistance to inappropriate pathogens that enter by direct penetration. Plant Cell. 2006;18(3):731-46.

20. Lu X, Dittgen J, Piślewska-Bednarek M, Molina A, Schneider B, Svatoš A, et al. Mutant allele-specific uncoupling of PENETRATION3 functions reveals engagement of the ATP-binding cassette transporter in distinct tryptophan metabolic pathways. Plant Physiol. 2015;168(3):814-27.

21. Xu J, Meng J, Meng X, Zhao Y, Liu J, Sun T, et al. Pathogen-responsive MPK3 and MPK6 reprogram the biosynthesis of indole glucosinolates and their derivatives in Arabidopsis immunity. Plant Cell. 2016;28(5):1144-62.

22. Kobae $Y$, Sekino T, Yoshioka H, Nakagawa T, Martinoia E, Maeshima M. Loss of AtPDR8, a plasma membrane ABC transporter of Arabidopsis thaliana, causes hypersensitive cell death upon pathogen infection. Plant Cell Physiol. 2006;47(3):309-18.

23. Lipka V, Dittgen J, Bednarek P, Bhat R, Wiermer M, Stein M, et al. Pre- and postinvasion defenses both contribute to nonhost resistance in Arabidopsis. Science. 2005;310(5751):1180-3.

24. Kopischke M, Westphal L, Schneeberger K, Clark R, Ossowski S, Wewer V, et al. Impaired sterol ester synthesis alters the response of Arabidopsis thaliana to Phytophthora infestans. Plant J. 2013;73(3):456-68.

25. Geissler K, Eschen-Lippold L, Naumann K, Schneeberger K, Weigel D, Scheel $D$, et al. Mutations in EDR1 gene alter the response of Arabidopsis thaliana to Phytophthora infestans and the bacterial PAMPs, flg22 and elf18. Mol Plant-Microbe Interact. 2015;28(2):122-33.

26. Bednarek P, Piślewska-Bednarek M, Ver Loren van Themaat E, Maddula RK, Svatoš A, Schulze-Lefert P. Conservation and clade-specific diversification of pathogen-inducible tryptophan and indole glucosinolate metabolism in Arabidopsis thaliana relatives. New Phytol. 2011;192(3):713-26.

27. Piasecka A, Jedrzejczak-Rey N, Bednarek P. Secondary metabolites in plant innate immunity: conserved function of divergent chemicals. New Phytol. 2015;206(3):948-64.

28. Mithen R, Bennett R, Marquez J. Glucosinolate biochemical diversity and innovation in the Brassicales. Phytochemistry. 2010;71(17-18):2074-86.

29. Bednarek P. Chemical warfare or modulators of defence responses - the function of secondary metabolites in plant immunity. Curr Opin Plant Biol. 2012;15(4):407-14.

30. Bednarek P. Sulfur-containing secondary metabolites from Arabidopsis thaliana and other Brassicaceae with function in plant immunity. ChemBioChem. 2012;13(13):1846-59.

31. Wang YAN, Bouwmeester K, van de Mortel JE, Shan W, Govers F. A novel Arabidopsis-oomycete pathosystem: differential interactions with Phytophthora capsici reveal a role for camalexin, indole glucosinolates and salicylic acid in defence. Plant Cell Environ. 2013;36(6):1192-203.
32. Schlaeppi K, Abou-Mansour E, Buchala A, Mauch F. Disease resistance of Arabidopsis to Phytophthora brassicae is established by the sequential action of indole glucosinolates and camalexin. Plant J. 2010;62(5):840-51.

33. Pedras MSC, Hossain S, Snitynsky RB. Detoxification of cruciferous phytoalexins in Botrytis cinerea: spontaneous dimerization of a camalexin metabolite. Phytochemistry. 2011;72(2-3):199-206.

34. Pedras MSC, Abdoli A. Metabolism of the phytoalexins camalexins, their bioisosteres and analogues in the plant pathogenic fungus Alternaria brassicicola. Bioorg Med Chem. 2013;21(15):4541-9.

35. Pedras MSC, Ahiahonu PWK. Probing the phytopathogenic stem rot fungus with phytoalexins and analogues: unprecedented glucosylation of camalexin and 6-methoxycamalexin. Bioorg Med Chem. 2002;10(10):3307-12.

36. Vlot AC, Dempsey DMA, Klessig DF. Salicylic acid, a multifaceted hormone to combat disease. Annu Rev Phytopathol. 2009;47(1):177-206.

37. Boatwright JL, Pajerowska-Mukhtar K. Salicylic acid: an old hormone up to new tricks. Mol Plant Pathol. 2013;14(6):623-34.

38. Tsuda K, Mine A, Bethke G, Igarashi D, Botanga CJ, Tsuda Y, et al. Dual regulation of gene expression mediated by extended MAPK activation and salicylic acid contributes to robust innate immunity in Arabidopsis thaliana. PLoS Genet. 2013;9(12):e1004015.

39. Mishina TE, Zeier J. Pathogen-associated molecular pattern recognition rather than development of tissue necrosis contributes to bacterial induction of systemic acquired resistance in Arabidopsis. Plant J. 2007:50(3):500-13.

40. Tsuda K, Sato M, Glazebrook J, Cohen JD, Katagiri F. Interplay between MAMPtriggered and SA-mediated defense responses. Plant J. 2008;53(5):763-75.

41. Nawrath C, Métraux J-P. Salicylic acid induction-deficient mutants of Arabidopsis express $P R-2$ and $P R-5$ and accumulate high levels of camalexin after pathogen inoculation. Plant Cell. 1999;11(8):1393-404.

42. An C, Mou Z. Non-host defense response in a novel ArabidopsisXanthomonas citri subsp. citri pathosystem. PLoS ONE. 2012;7(1):e31130.

43. Tanaka S, Han X, Kahmann R. Microbial effectors target multiple steps in the salicylic acid production and signaling pathway. Front Plant Sci. 2015;6:349.

44. Whisson SC, Boevink PC, Moleleki L, Avrova AO, Morales JG, Gilroy EM, et al. A translocation signal for delivery of oomycete effector proteins into host plant cells. Nature. 2007;450(7166):115-8.

45. Caten CE, Jinks JL. Spontaneous variability of single isolates of Phytophthora infestans. I. Cultural variation. Can J Bot. 1968;46(4):329.

46. Kamoun S, van West P, Vleeshouwers VGAA, de Groot KE, Govers F. Resistance of Nicotiana benthamiana to Phytophthora infestans is mediated by the recognition of the elicitor protein INF1. Plant Cell. 1998;10(9):141325

47. Kemen E, Gardiner A, Schultz-Larsen T, Kemen AC, Balmuth AL, RobertSeilaniantz A, et al. Gene gain and loss during evolution of obligate parasitism in the white rust pathogen of Arabidopsis thaliana. PLoS Biol. 2011;9(7):e1001094.

48. Xiao S, Ellwood S, Calis O, Patrick E, Li T, Coleman M, et al. Broad-spectrum mildew resistance in Arabidopsis thaliana mediated by RPW8. Science. 2001; 291(5501):118-20.

49. Caillaud M-C, Asai S, Rallapalli G, Piquerez S, Fabro G, Jones JDG. A downy mildew effector attenuates salicylic acid-triggered immunity in Arabidopsis by interacting with the host mediator complex. PLoS Biol. 2013;11(12):e1001732.

50. Asai S, Rallapalli G, Piquerez SJM, Caillaud M-C, Furzer OJ, Ishaque N, et al. Expression profiling during Arabidopsis/downy mildew interaction reveals a highly-expressed effector that attenuates responses to salicylic acid. PLoS Pathog. 2014;10(10):e1004443.

51. Stefanato FL, Abou-Mansour E, Buchala A, Kretschmer M, Mosbach A, Hahn $M$, et al. The ABC transporter BcatrB from Botrytis cinerea exports camalexin and is a virulence factor on Arabidopsis thaliana. Plant J. 2009;58(3):499-510.

52. Schoonbeek H, Del Sorbo G, De Waard MA. The ABC transporter BcatrB affects the sensitivity of Botrytis cinerea to the phytoalexin resveratrol and the fungicide fenpiclonil. Mol Plant-Microbe Interact. 2001;14(4):562-71.

53. Kretschmer M, Leroch M, Mosbach A, Walker A-S, Fillinger S, Mernke D, et al. Fungicide-driven evolution and molecular basis of multidrug resistance in field populations of the grey mould fungus Botrytis cinerea. PLoS Pathog. 2009:5(12):e1000696.

54. Leroch M, Mernke D, Koppenhoefer D, Schneider P, Mosbach A, Doehlemann $\mathrm{G}$, et al. Living colors in the gray mold pathogen Botrytis cinerea: Codon-optimized genes encoding green fluorescent protein and mCherry, which exhibit bright fluorescence. Appl Environ Microbiol. 2011;77(9):2887-97. 
55. Kover PX, Valdar W, Trakalo J, Scarcelli N, Ehrenreich IM, Purugganan $M D$, et al. A multiparent advanced generation inter-cross to fine-map quantitative traits in Arabidopsis thaliana. PLoS Genet. 2009;5(7): e1000551.

56. Rallapalli G, Kemen E, Robert-Seilaniantz A, Segonzac C, Etherington G, Sohn $\mathrm{K}$, et al. EXPRSS: an Illumina based high-throughput expressionprofiling method to reveal transcriptional dynamics. BMC Genomics. 2014;15:341.

57. Edgar R, Domrachev M, Lash AE. Gene Expression Omnibus: NCBI gene expression and hybridization array data repository. Nucleic Acids Res. 2002;30(1):207-10.

58. The Arabidopsis Information Resource. www.arabidopsis.org. Accessed 3 Mar 2011.

59. Langmead B, Trapnell C, Pop M, Salzberg S. Ultrafast and memory-efficient alignment of short DNA sequences to the human genome. Genome Biol. 2009;10(3):R25.

60. Novocraft. http://www.novocraft.com

61. shyamrallapalli/publication_data. 2015. https://github.com/shyamrallapalli/ publication_data/tree/master/2015_dprince_albugo.

62. R Core Team. R: A language and environment for statistical computing. Vienna, Austria: R Foundation for Statistical Computing; 2015.

63. Gentleman R, Carey V, Bates D, Bolstad B, Dettling M, Dudoit S, et al. Bioconductor: open software development for computational biology and bioinformatics. Genome Biol. 2004;5(10):R80.

64. Robinson MD, McCarthy DJ, Smyth GK. edgeR: a Bioconductor package for differential expression analysis of digital gene expression data. Bioinformatics. 2010;26(1):139-40.

65. Benjamini Y, Hochberg Y. Controlling the false discovery rate: a practical and powerful approach to multiple testing. J R Stat Soc Ser B Methodol. 1995;57(1):289-300.

66. Wang D, Amornsiripanitch N, Dong X. A genomic approach to identify regulatory nodes in the transcriptional network of systemic acquired resistance in plants. PLoS Pathog. 2006;2(11):e123.

67. Goda H, Sasaki E, Akiyama K, Maruyama-Nakashita A, Nakabayashi K, Li W, et al. The AtGenExpress hormone and chemical treatment data set: experimental design, data evaluation, model data analysis and data access. Plant J. 2008;55(3):526-42.

68. Van der Does D, Leon-Reyes A, Koornneef A, Van Verk MC, Rodenburg N, Pauwels $L$, et al. Salicylic acid suppresses jasmonic acid signaling downstream of SCFCOI1-JAZ by targeting GCC promoter motifs via transcription factor ORA59. Plant Cell. 2013;25(2):744-61.

69. Public Research Centre for Health. Venn Diagram.

70. Du Z, Zhou X, Ling Y, Zhang Z, Su Z. agriGO: a GO analysis toolkit for the agricultural community. Nucleic Acids Res. 2010;38 suppl 2:W64-70.

71. Prince DC, Drurey C, Zipfel C, Hogenhout SA. The leucine-rich repeat receptor-like kinase brassinosteroid insensitive1-associated kinase1 and the cytochrome P450 phytoalexin deficient3 contribute to innate immunity to aphids in Arabidopsis. Plant Physiol. 2014;164(4):2207-19.

72. Czechowski T, Stitt M, Altmann T, Udvardi MK, Scheible W-R. Genome-wide identification and testing of superior reference genes for transcript normalization in Arabidopsis. Plant Physiol. 2005;139(1):5-17.

73. Vandesompele J, De Preter K, Pattyn F, Poppe B, Van Roy N, De Paepe A, et al. Accurate normalization of real-time quantitative RT-PCR data by geometric averaging of multiple internal control genes. Genome Biol. 2002;3(7):RESEARCH0034

74. Andersen $\mathrm{CL}$, Jensen JL, Ørntoft TF. Normalization of real-time quantitative reverse transcription-PCR data: a model-based variance estimation approach to identify genes suited for normalization, applied to bladder and colon cancer data sets. Cancer Res. 2004;64(15):5245-50.

75. Le Pape S. EasyqpcR: EasyqpcR for easy analysis of real-time PCR data at IRTOMIT-INSERM U1082. R package. 2012. https://www.bioconductor.org/ packages/release/bioc/html/EasyqpcR.html. Accessed 9 Sept 2015.

76. Rieu I, Powers SJ. Real-time quantitative RT-PCR: design, calculations, and statistics. Plant Cell. 2009:21(4):1031-3

77. Kliebenstein DJ, Lambrix VM, Reichelt M, Gershenzon J, Mitchell-Olds T. Gene duplication in the diversification of secondary metabolism: tandem 2-oxoglutarate-dependent dioxygenases control glucosinolate biosynthesis in Arabidopsis. Plant Cell. 2001;13(3):681-93.

78. Brown PD, Tokuhisa JG, Reichelt M, Gershenzon J. Variation of glucosinolate accumulation among different organs and developmental stages of Arabidopsis thaliana. Phytochemistry. 2003;62(3):471-81.
79. Reichelt M, Brown PD, Schneider B, Oldham NJ, Stauber E, Tokuhisa J, et al. Benzoic acid glucosinolate esters and other glucosinolates from Arabidopsis thaliana. Phytochemistry. 2002;59(6):663-71.

80. Fiebig HJ, Arens M. Glucosinolates (HPLC method) - Survey by a working party of the DGF, 128th report - German standard methods for investigation of fats, fatty products, tensides and related materials, 98th report - analysis of fat raw-materials 12. Fett Wissenschaft Technol Fat Sci Technol. 1992;94(6):199-203.

81. Schoonbeek, H-j, Jacquat-Bovet A-C, Mascher F, Métraux J-P. Oxalatedegrading bacteria can protect Arabidopsis thaliana and crop plants against Botrytis cinerea. Mol Plant-Microbe Interact. 2007;20(12):1535-44.

82. X-gluc Direct. http://www.X-gluc.com.

83. Spex Sample Prep. http://www.spexsampleprep.com.

84. Jefferson RA, Kavanagh TA, Bevan MW. GUS fusions - beta-glucuronidase as a sensitive and versatile gene fusion marker in higher-plants. EMBO J. 1987;6(13):3901-7.

85. Gachon C, Saindrenan P. Real-time PCR monitoring of fungal development in Arabidopsis thaliana infected by Alternaria brassicicola and Botrytis cinerea. Plant Physiol Biochem. 2004;42(5):367-71.

86. Schneider CA, Rasband WS, Eliceiri KW. NIH Image to ImageJ: 25 years of image analysis. Nat Meth. 2012;9(7):671-5.

87. RStudio. RStudio: Integrated development environment for R. Boston, MA: RStudio, Inc.; 2015.

88. Clay NK, Adio AM, Denoux C, Jander G, Ausubel FM. Glucosinolate metabolites required for an Arabidopsis innate immune response. Science. 2009;323(5910):95-101.

89. Guo R, Qian H, Shen W, Liu L, Zhang M, Cai C, et al. BZR1 and BES1 participate in regulation of glucosinolate biosynthesis by brassinosteroids in Arabidopsis. J Exp Bot. 2013;64(8):2401-12.

90. Sønderby IE, Hansen BG, Bjarnholt N, Ticconi C, Halkier BA, Kliebenstein DJ. A systems biology approach identifies a R2R3 MYB gene subfamily with distinct and overlapping functions in regulation of aliphatic glucosinolates. PLoS ONE. 2007;2(12):e1322.

91. Barth C, Jander G. Arabidopsis myrosinases TGG1 and TGG2 have redundant function in glucosinolate breakdown and insect defense. Plant J. 2006;46(4):549-62.

92. Ferrari S, Plotnikova JM, De Lorenzo G, Ausubel FM. Arabidopsis local resistance to Botrytis cinerea involves salicylic acid and camalexin and requires EDS4 and PAD2, but not SID2, EDS5 or PAD4. Plant J. 2003:35(2):193-205.

93. Kliebenstein DJ, Rowe HC, Denby KJ. Secondary metabolites influence Arabidopsis/Botrytis interactions: variation in host production and pathogen sensitivity. Plant J. 2005:44(1):25-36.

94. Pieterse CMJ, Van der Does D, Zamioudis C, Leon-Reyes A, Van Wees SCM. Hormonal modulation of plant immunity. Annu Rev Cell Dev Biol. 2012; 28(1):489-521.

95. Weigel RR, Bäuscher C, Pfitzner AJP, Pfitzner UM. NIMIN-1, NIMIN-2 and NIMIN-3, members of a novel family of proteins from Arabidopsis that interact with NPR1/NIM1, a key regulator of systemic acquired resistance in plants. Plant Mol Biol. 2001;46(2):143-60.

96. Wildermuth MC, Dewdney J, Wu G, Ausubel FM. Isochorismate synthase is required to synthesize salicylic acid for plant defence. Nature. 2001; 414(6863):562-5.

97. Van Wees SCM, Glazebrook J. Loss of non-host resistance of Arabidopsis NahG to Pseudomonas syringae pv. phaseolicola is due to degradation products of salicylic acid. Plant J. 2003;33(4):733-42.

98. Ruhe J, Agler MT, Placzek A, Kramer K, Finkemeier I, Kemen EM. Obligate biotroph pathogens of the genus Albugo are better adapted to active host defense compared to niche competitors. Front Plant Sci. 2016;7:820.

99. Bilgin DD, Zavala JA, Zhu JIN, Clough SJ, Ort DR, DeLucia EH. Biotic stress globally downregulates photosynthesis genes. Plant Cell Environ. 2010;33(10):1597-613.

100. Sanchez-Vallet A, Ramos B, Bednarek P, López G, Piślewska-Bednarek M, SchulzeLefert $P$, et al. Tryptophan-derived secondary metabolites in Arabidopsis thaliana confer non-host resistance to necrotrophic Plectosphaerella cucumerina fungi. Plant J. 2010;63(1):115-27.

101. Bak S, Tax FE, Feldmann KA, Galbraith DW, Feyereisen R. CYP83B1, a cytochrome $\mathrm{P} 450$ at the metabolic branch point in auxin and indole glucosinolate biosynthesis in Arabidopsis. Plant Cell. 2001;13(1):101-11.

102. Smolen G, Bender J. Arabidopsis cytochrome P450 cyp83B1 mutations activate the tryptophan biosynthetic pathway. Genetics. 2002;160(1):323-32.

103. Zhao Y, Hull AK, Gupta NR, Goss KA, Alonso J, Ecker JR, et al. Trp-dependent auxin biosynthesis in Arabidopsis: involvement of cytochrome P450s CYP79B2 and CYP79B3. Genes Dev. 2002;16(23):3100-12. 
104. Glawischnig E, Hansen BG, Olsen CE, Halkier BA. Camalexin is synthesized from indole-3-acetaldoxime, a key branching point between primary and secondary metabolism in Arabidopsis. Proc Natl Acad Sci U S A. 2004; 101(21):8245-50

105. Hiruma K, Fukunaga S, Bednarek P, Piślewska-Bednarek M, Watanabe S Narusaka $Y$, et al. Glutathione and tryptophan metabolism are required for Arabidopsis immunity during the hypersensitive response to hemibiotrophs. Proc Natl Acad Sci. 2013;110(23):9589-94.

106. Rajniak J, Barco B, Clay NK, Sattely ES. A new cyanogenic metabolite in Arabidopsis required for inducible pathogen defence. Nature. 2015; 525(7569):376-9.

107. Dobritzsch M, Lübken T, Eschen-Lippold L, Gorzolka K, Blum E, Matern A, et al. MATE transporter-dependent export of hydroxycinnamic acid amides. Plant Cell. 2016;28(2):583-96.

108. Djamei A, Schipper K, Rabe F, Ghosh A, Vincon V, Kahnt J, et al. Metabolic priming by a secreted fungal effector. Nature. 2011;478(7369):395-8.

109. Tanaka S, Brefort T, Neidig N, Djamei A, Kahnt J, Vermerris W, et al. A secreted Ustilago maydis effector promotes virulence by targeting anthocyanin biosynthesis in maize. elife. 2014;3:e01355.

110. Roetschi A, Si-Ammour A, Belbahri L, Mauch F, Mauch-Mani B. Characterization of an Arabidopsis-Phytophthora Pathosystem: resistance requires a functional PAD2 gene and is independent of salicylic acid, ethylene and jasmonic acid signalling. Plant J. 2001;28(3):293-305.

111. Fabro G, Steinbrenner J, Coates M, Ishaque N, Baxter L, Studholme DJ, et al. Multiple candidate effectors from the oomycete pathogen Hyaloperonospora arabidopsidis suppress host plant immunity. PLoS Pathog. 2011;7(11):e1002348.

112. Bos JIB, Prince D, Pitino M, Maffei ME, Win J, Hogenhout SA. A functional genomics approach identifies candidate effectors from the aphid species Myzus persicae (Green Peach Aphid). PLoS Genet. 2010;6(11):e1001216.

113. Buxdorf $K$, Yaffe $H$, Barda O, Levy M. The effects of glucosinolates and their breakdown products on necrotrophic fungi. PLOS ONE. 2013;8(8):e70771.

114. Frerigmann $\mathrm{H}$, Gigolashvili T. MYB34, MYB51, and MYB122 distinctly regulate indolic glucosinolate biosynthesis in Arabidopsis thaliana. Mol Plant. 2014;7(5):814-28.

\section{Submit your next manuscript to BioMed Central and we will help you at every step:}

- We accept pre-submission inquiries

- Our selector tool helps you to find the most relevant journal

- We provide round the clock customer support

- Convenient online submission

- Thorough peer review

- Inclusion in PubMed and all major indexing services

- Maximum visibility for your research

Submit your manuscript at www.biomedcentral.com/submit 